\title{
Incoherent effects of space charge and sum resonances on particle beams in a storage ring
}

\author{
G. Franchetti@ \\ GSI Helmholtzzentrum für Schwerionenforschung GmbH, Planckstrasse 1, 64291 Darmstadt, Germany \\ and Goethe Universität Frankfurt, Max-von-Laue-Straße 7, 60438 Frankfurt am Main, Germany
}

(Received 4 August 2018; published 25 November 2019)

\begin{abstract}
In the past years, several studies, numerical and experimental, have been carried out for enlightening the effect of space charge and resonances on particle beams stored in accelerators. The last effort of this quest focused on the third-order coupled resonances, emphasizing the existence of the periodic crossing of fixed lines induced by space charge. In this article, the theory of nonlinear sum resonances in coasting beams in the presence of space charge is presented and applied to unravel the key features of the four-dimensional coupled dynamics in high-intensity bunched beams.
\end{abstract}

DOI: 10.1103/PhysRevAccelBeams.22.114201

\section{THE INCOHERENT SPACE CHARGE REGIME}

Collective effects in a non-neutral plasma as a beam in a particle accelerator are related to the Debye length $\lambda \propto \sqrt{\overline{v_{\mathrm{th}}^{2}} / n}$, where $\overline{v_{\mathrm{th}}^{2}}$ is the rms square "thermal" component of the velocity of the particles and $n$ the particle density $[1,2]$. The length $\lambda$ is characteristic of the collective motion of charged particles: Local charge perturbations in plasma are shielded within $\lambda$. If $\lambda$ is larger than the average interparticle distance, space charge can be treated as a smoothly applied force. If also $\lambda$ is larger than the beam sizes, single-particle behavior dominates over the collective dynamics (see Ref. [1]). Next, a coasting beam with particle density

$$
n(x, y, s)=\frac{N}{\pi \sigma_{x}(s) \sigma_{y}(s)} F^{\prime}\left(\frac{x^{2}}{\sigma_{x}^{2}(s)}+\frac{y^{2}}{\sigma_{y}^{2}(s)}\right)
$$

is considered, where $N$ is the particle line density and $\sigma_{\chi}$ with $\chi=x, y$ is the rms beam size matched to the accelerator optics. The function $F^{\prime}(t)=d F(t) / d t$ characterizes the type of beam distribution and has the maximum at $t=0$. In Appendix A, we show the most common distributions. The rms thermal velocity is given by $\overline{v_{\chi}^{2} \text {,th }}(s)=v_{0}^{2} \epsilon_{\chi} / \beta_{\chi}(s)$, where $v_{0}$ is the particles velocity, $\epsilon_{\chi}$ is the rms beam emittance, and $\beta_{\chi}(s)$ is the optics function. In order to assess the relevance of the incoherent effects of space charge, using the argument of Ref. [1], we define the parameter $\mathcal{I}_{\chi}^{2}$ as the average of $\lambda_{S, \chi}^{2}(s) / \sigma_{\chi}^{2}(s)$ along the accelerator circumference,

Published by the American Physical Society under the terms of the Creative Commons Attribution 4.0 International license. Further distribution of this work must maintain attribution to the author(s) and the published article's title, journal citation, and DOI.
$\lambda_{S, \chi}(s)$ being the smallest transverse Debye length at location $s$. For particle distributions defined by Eq. (1), we find

$$
\mathcal{I}_{\chi} \gtrsim \mathcal{I}_{\chi, l}=\sqrt{\frac{\bar{\sigma}_{\bar{\chi}}}{\bar{\sigma}_{x}+\bar{\sigma}_{y}}} \frac{1+\frac{\Delta Q_{\chi}}{Q_{\chi 0}}}{\sqrt{1-\left(1+\frac{\Delta Q_{\chi}}{Q_{\chi 0}}\right)^{2}}},
$$

where $\bar{\chi}=y, x$ for $\chi=x, y, \bar{\sigma}_{\chi}=\left\{\left[R /\left(Q_{\chi 0}+\Delta Q_{\chi}\right)\right] \epsilon_{\chi}\right\}^{1 / 2}$, $R$ is the average accelerator radius, $\Delta Q_{\chi}$ is the maximum incoherent space charge tune shift (negative), and $Q_{\chi 0}$ is the machine tune. The calculation of $\Delta Q_{\chi}$ for an arbitrary intensity and in the limit of a small intensity is described in Appendix A. In Appendix B is described instead the derivation of Eq. (2), including the approximations adopted. Equation (2) shows that rms equivalent beams with the same $\Delta Q_{\chi} / Q_{\chi 0}$ have in good approximation the same $\mathcal{I}_{\chi, l}$. This equation is exact for a constant focusing channel. If $\mathcal{I}_{\chi}<1$ realistic stationary beam distributions are nonuniform, and their evolution is described by the Vlasov equation. However, only the evolution of KV distributions and their linearized perturbations can be solved analytically with a Vlasov analysis [3]. The situation becomes simpler in the limit $\mathcal{I}_{\chi} \simeq 0$ where the beam distribution becomes uniform in space and space charge forces become linear [1]. The larger the $\mathcal{I}_{\chi}$, the more direct space charge forces on beam particles act incoherently and for stationary beam distributions can be treated as external forces. Although the methods presented in this work apply to a large class of beam distributions, we mainly focus on a Gaussian distribution. Therefore, we tentatively define the regime of "incoherent" space charge when $\mathcal{I}_{\chi, l}>3$, assuming the "edge" of a Gaussian beam at 3 times the rms size.

Figure 1 shows an example of a particle distribution of a beam with $\mathcal{I}=\mathcal{I}_{x, l}=\mathcal{I}_{y, l}=0.1$ at two instants during the 

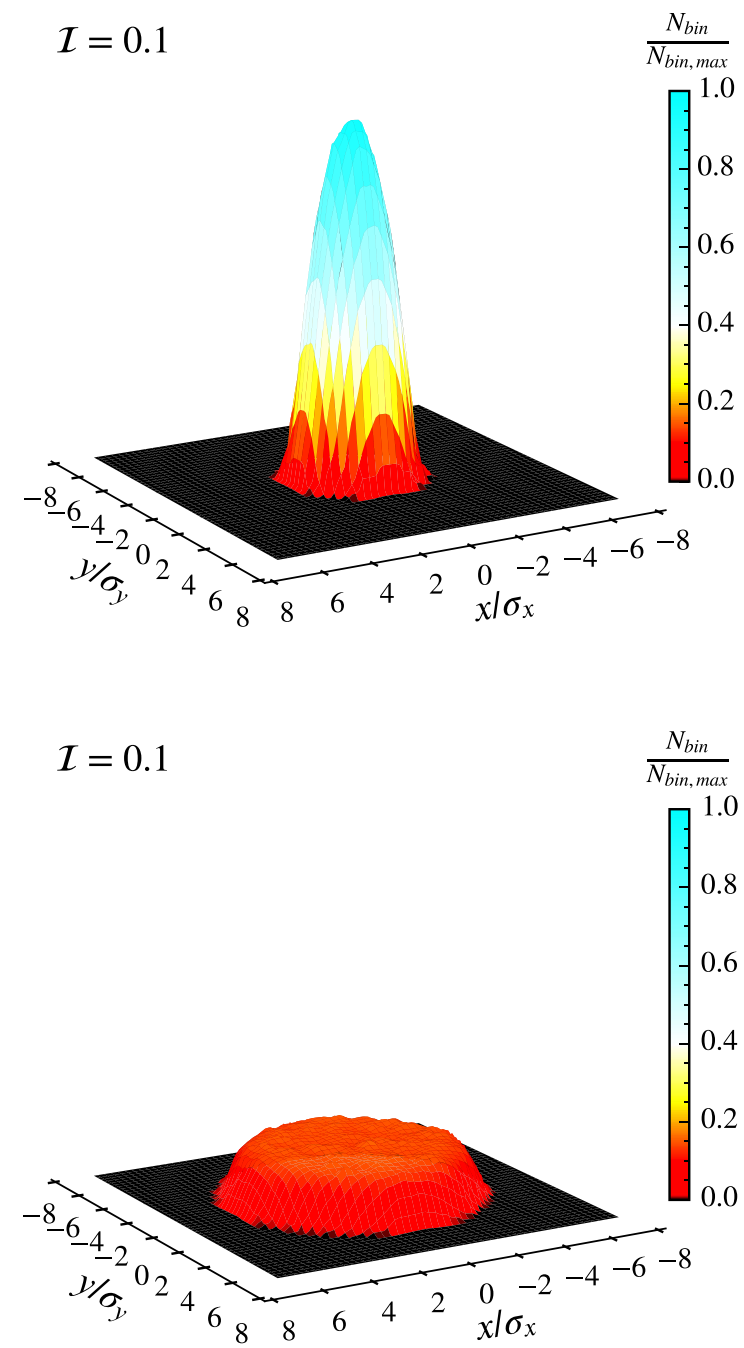

FIG. 1. A water bag beam is rms matched to a uniform focusing structure, and its intensity is strong enough to create a Debye length smaller than the rms beam sizes. The picture on top shows the initial particle distribution. The picture on the bottom shows the particle distribution at an instant during transport. It is visible that the "type" of beam distribution has changed from WB to uniform, highlighting a space-charge-driven collective dynamics of particles in the beam. Both histograms are normalized with the maximum bin particles of the initial particle distribution.

transport. The evolution is computed via particle-in-cell simulations. The initial particle distribution is an axisymmetric water bag (WB) and is rms matched with a uniform focusing channel with tunes $Q_{x 0}=Q_{y 0}=3.55$. The beam intensity is set to create an rms KV equivalent tune shift of $\Delta Q_{x}=\Delta Q_{y}=-3.06$. The tracking of this particle distribution shows that the beam undergoes oscillations, as the rms equivalent matching does not account for all space charge effects. Figure 1(top) shows the initial particle distribution, whereas the bottom picture shows the particle distribution after a phase advance of $43.4^{\circ}$ in the bare lattice, equivalent to $6.0^{\circ}$ of rms equivalent depressed phase advance. It is visible that the beam distribution in Fig. 1 (bottom) has a different character, as it has evolved from a WB to a spatially uniform distribution in the course of the mismatch oscillations. Similar simulations performed for $\mathcal{I}>0.67$ show that, although the beam undergoes mismatch oscillations, the character of the initial particle distribution is much better preserved, underlining a regime of incoherent space charge dynamics.

For axisymmetric beams, Eq. (2) indicates that if $\left|\Delta Q_{\chi}\right| / Q_{\chi 0}<0.0266$, space charge forces act in the incoherent regime, so the Coulomb and self-fields may be approximated as arising from a stationary particle distribution matched with the accelerator optics. This approach is what in past studies has been named as the frozen space charge. It also assumes that the beam distribution remains stationary; hence, the effects of the accelerator, as arising from machine nonlinearities or from beam coupling with the environment, are considered negligible. Dynamical effects induced by beam loss are neglected as well. For Gaussian beam distributions, these requirements are also satisfied because of the absence of coherent resonances [4].

Another reason to develop space charge frozen models is that they may also help in validating new particle-in-cell algorithms disentangling intrinsic noise heating effects from the dynamics [5-9].

\section{SUM RESONANCES AND INCOHERENT SPACE CHARGE}

The theory of resonances in accelerators was developed in the 1950s [10,11] based on a perturbative approach to the Hamiltonian mechanics which lead to the development of driving term methods to measure and correct resonances [12]. An extension of this approach uses normal form methods developed after the advent of computers and numerical physics $[13,14]$. In the absence of space charge, coupled sum resonances create a particular pattern of orbits in phase space called fixed lines [15-17]. Although known for a long time (earlier references trace to the 1980s $[18,19])$, but also more recently studied in Ref. [20,21], fixed lines have been regarded as a mathematical curiosity with no significant impact on beam quality, as they can be avoided with a proper choice of the accelerator working point. However, the recent study in Ref. [22] has shown that fixed lines play a fundamental role in determining the dynamics of halo formation in high-intensity bunched beams. Next, adopting a perturbative approach, the theory of sum coupled resonances including a frozen model of space charge modeling a Gaussian beam will be discussed. Alternatively, but for 1D resonances, a normal form approach with frozen space charge is discussed in Ref. [23].

\section{A. Hamiltonian and space charge potential}

The single-particle Hamiltonian is composed by a quadratic form $H_{0}$ determined by the sequence of accelerator quadrupoles 


$$
H_{0}=\frac{1}{2}\left(x^{\prime 2}+y^{\prime 2}\right)+\frac{1}{2}\left[k_{x}(s) x+k_{y}(s) y\right]
$$

and by the perturbation term $H_{1}(x, y, s)=V_{m}(x, y, s)+$ $V_{\mathrm{sc}}(x, y, s)$ formed by the potential of the nonlinear errors in magnets $V_{m}(x, y, s)$ and of the frozen space charge $V_{\mathrm{sc}}(x, y, s)$. The frozen model approximation of the space charge potential has the analytic form

$V_{\mathrm{sc}}(x, y, s)=-\frac{K}{2} \int_{0}^{\infty} \frac{F(T(x, y, s, t))-F(0)}{\left[\sigma_{x}^{2}(s)+t\right]^{1 / 2}\left[\sigma_{y}^{2}(s)+t\right]^{1 / 2}} d t$,

with $T(x, y, s, t)=x^{2} /\left[\sigma_{x}^{2}(s)+t\right]+y^{2} /\left[\sigma_{y}^{2}(s)+t\right]$ and $K$ the beam perveance [1]. In the following, we will discuss only a Gaussian beam which is characterized by $F(t)=-\exp (-t / 2)$. In fact, by replacing this $F(t)$ in Eq. (1), we find the expected particle distribution

$$
n(x, y, s)=\frac{N}{2 \pi \sigma_{x}(s) \sigma_{y}(s)} \exp \left[-\frac{x^{2}}{2 \sigma_{x}^{2}(s)}-\frac{y^{2}}{2 \sigma_{y}^{2}(s)}\right] .
$$

\section{B. Scaled Hamiltonian}

We now apply the following canonical transformation:

$$
\hat{x}=\frac{x}{\sqrt{\epsilon_{x}}}, \quad \hat{p}_{x}=\sqrt{\epsilon_{x}} x^{\prime}, \quad \hat{y}=\frac{y}{\sqrt{\epsilon_{x}}}, \quad \hat{p}_{y}=\sqrt{\epsilon_{x}} y^{\prime}
$$

and add the symbol ' to all variables in this system of coordinates. The quantity $\epsilon_{x}$ is the horizontal $\mathrm{rms}$ beam emittance. The Hamiltonian becomes $\hat{H}=\hat{H}_{0}+\hat{H}_{1}$, where

$$
\hat{H}_{0}=\frac{1}{2 \epsilon_{x}} \hat{p}_{x}^{2}+\frac{1}{2 \epsilon_{x}} \hat{p}_{y}^{2}+\frac{1}{2} k_{x}(s) \epsilon_{x} \hat{x}^{2}+\frac{1}{2} k_{y}(s) \epsilon_{x} \hat{y}^{2}
$$

and $\hat{H}_{1}=\hat{V}_{\mathrm{sc}}(\hat{x}, \hat{y}, s)+\hat{V}_{m}(\hat{x}, \hat{y}, s)$ with the two potentials $\hat{V}_{\mathrm{sc}}(\hat{x}, \hat{y}, s)=V_{\mathrm{sc}}\left(\sqrt{\epsilon_{x}} \hat{x}, \sqrt{\epsilon_{x}} \hat{y}, s\right)$ and $\hat{V}_{m}(\hat{x}, \hat{y}, s)=$ $V_{m}\left(\sqrt{\epsilon_{x}} \hat{x}, \sqrt{\epsilon_{x}} \hat{y}, s\right)$. The solution of the equations of motion for the unperturbed Hamiltonian $H_{0}$ is expressed in a modified Courant-Snyder form: $\chi(s)=$ $\sqrt{\beta_{\chi}(s) \hat{a}_{\chi} \epsilon_{x}} \cos \left[\psi_{\chi}(s)+\hat{\varphi}_{\chi}\right]$ (we recall that $\chi=x, y$ ). With this definition, the usual single-particle emittances are scaled to the horizontal rms beam emittance and are called $\hat{a}_{\chi}$. The relation of the phase $\hat{\varphi}_{\chi}$ of the modified solution with the standard form is $\phi_{\chi}=\hat{\varphi}_{\chi}+\pi / 2$.

\section{Full solution}

The dynamics of particles under the full Hamiltonian $\hat{H}_{0}+\hat{H}_{1}$ is derived by keeping the Courant-Snyder form but allowing the quantities $\hat{a}_{\chi}$ and $\hat{\varphi}_{\chi}$ to vary with $s$. Therefore, the quantities $\hat{a}_{\chi}$ and $\hat{\varphi}_{\chi}$ become dynamical coordinates controlled by the Hamiltonian $\hat{H}_{1}$. The form of $\hat{H}_{1}$ is obtained replacing $\chi$ with the Courant-Snyder form; hence,

$$
\begin{aligned}
\hat{H}_{1}\left(\hat{a}_{x}, \hat{\varphi}_{x}, \hat{a}_{y}, \hat{\varphi}_{y}, s\right)= & \hat{V}_{m}\left[\hat{x}\left(\hat{a}_{x}, \hat{\varphi}_{x}\right), \hat{y}\left(\hat{a}_{y}, \hat{\varphi}_{y}\right), s\right] \\
& +\hat{V}_{\mathrm{sc}}\left[\hat{x}\left(\hat{a}_{x}, \hat{\varphi}_{x}\right), \hat{y}\left(\hat{a}_{y}, \hat{\varphi}_{y}\right), s\right]
\end{aligned}
$$

with $\hat{\chi}\left(\hat{a}_{\chi}, \hat{\varphi}_{\chi}\right)=\sqrt{\beta_{\chi}(s) \hat{a}_{\chi}} \cos \left[\psi_{\chi}(s)+\hat{\varphi}_{\chi}\right]$. This procedure leads to a new set of four canonical equations, determined by the Hamiltonian $\hat{H}_{1}$, namely,

$$
\hat{a}_{\chi}^{\prime}=-\frac{2}{\epsilon_{x}} \frac{\partial \hat{H}_{1}}{\partial \hat{\varphi}_{\chi}}, \quad \hat{\varphi}_{\chi}^{\prime}=\frac{2}{\epsilon_{x}} \frac{\partial \hat{H}_{1}}{\partial \hat{a}_{\chi}} .
$$

It can be shown that the Hamiltonian $\hat{H}_{1}\left(\hat{a}_{x}, \hat{\varphi}_{x}, \hat{a}_{y}, \hat{\varphi}_{y}, s\right)$ is expressed as a harmonics series in which each term of the series is an oscillating function whose frequency is a combination of the machine tunes and the harmonics number $m$ of the machine nonlinear errors along the accelerator. In Appendix C, we show the form of $\hat{H}_{1}$.

\section{Slowly varying Hamiltonian}

By setting the machine tunes $Q_{x 0}$ and $Q_{y 0}$ such that the frequency of some harmonics becomes "slow," which happens when $Q_{x 0}$ and $Q_{y 0}$ are set close $N_{x} Q_{x}+N_{y} Q_{y}=$ $m$ for the excited resonance, the system of canonical equations acquires a dynamics in which the average motion is controlled mainly by the "slow harmonics" $[24,25]$. Then the corresponding slowly varying Hamiltonian $\hat{H}_{1 s}$ is taken as the representative of the original system. Clearly, this is a substantial approximation, which nevertheless for weak errors and small distances from the resonance under consideration yields acceptable modeling of the dynamics. The form of this Hamiltonian is discussed in Appendix D.

\section{E. Time-independent slowly varying Hamiltonian}

Next, the canonical transformation of generating function

$$
F_{2}\left(\hat{\varphi}_{x}, \tilde{a}_{x}, \hat{\varphi}_{y}, \tilde{a}_{y}\right)=\tilde{a}_{x} \hat{\varphi}_{x}+\tilde{a}_{y} \hat{\varphi}_{y}+\left(\tilde{a}_{x} t_{x}+\tilde{a}_{y} t_{y}\right) \frac{\Delta_{r 0}}{R} s
$$

is applied to remove the time dependence from the slowly varying Hamiltonian: The parameters $t_{x}$ and $t_{y}$, are subject to the condition $N_{x} t_{x}+N_{y} t_{y}=1$. As result, there are infinite canonical transformations, each identified by $t_{x}$, able to create a new set of canonical variables $\tilde{a}_{x}, \tilde{\varphi}_{x}, \tilde{a}_{y}, \tilde{\varphi}_{y}$ which have the property $\tilde{a}_{x}=\hat{a}_{x}, \tilde{a}_{y}=\hat{a}_{y}$, and remove the time dependency; the dynamics of these variables is now controlled by the timeindependent (slowly varying) Hamiltonian

$$
\begin{aligned}
\tilde{H}_{1}= & \frac{4 \rho_{s}}{R} \tilde{a}_{x}^{n_{x} / 2} \tilde{a}_{y}^{n_{y} / 2} \cos \left[N_{x} \tilde{\varphi}_{x}+N_{y} \tilde{\varphi}_{y}+\alpha\right] \\
& +\left(\tilde{a}_{x} t_{x}+\tilde{a}_{y} t_{y}\right) \frac{\Delta_{r 0}}{R}+\tilde{V}_{n r},
\end{aligned}
$$

with $\tilde{V}_{n r}=\hat{V}_{n r}$ (see Appendix D). The first term in Eq. (5) represents the resonance, and the coefficients $\rho_{s} \geq 0$ and $\alpha$ are the scaled amplitude and phase of the driving term, respectively. Here, we simplify the notation, setting 
$\rho_{s}=\rho_{n_{x}, n_{y}, j_{x}, j_{y},-m} R / \epsilon_{x}$ and $\alpha=\alpha_{n_{x}, n_{y}, j_{x}, j_{y},-m}$ of the single resonance excited. See Appendix D for a discussion on the driving terms from space charge and from magnets. In this work, we focus on resonances driven by magnets; however, the same approach can be applied to resonances driven by space charge. In Ref. [26], a study in this regime is presented for one-dimensional resonances. In Eq. (5), the only free parameter in addition to $t_{x}$ is the distance from the resonance $\Delta_{r 0}=N_{x} Q_{x 0}+N_{y} Q_{y 0}-m$, which plays a fundamental role in the dynamics. The slowly varying potential $\tilde{V}_{n r}$ is a function of the $\tilde{a}_{x}, \tilde{a}_{y}$ only and is formed by the contributions from all nonlinear components in magnets and space charge. From the potential in Eq. (3), it is found that

$$
\tilde{V}_{n r, s c}\left(\tilde{a}_{x}, \tilde{a}_{y}\right)=\frac{1}{2 \pi} \int_{0}^{2 \pi} d x \frac{1}{2 \pi} \int_{0}^{2 \pi} d y \frac{1}{L} \int_{0}^{L} d s \frac{2}{\epsilon_{x}} V_{\mathrm{sc}}\left[\cos (x) \sigma_{x}(s) \sqrt{\tilde{a}_{x}}, \cos (y) \sigma_{y}(s) \sqrt{\epsilon_{r} \tilde{a}_{y}}, s\right],
$$

with $\epsilon_{r}=\epsilon_{x} / \epsilon_{y}$ the beam emittance ratio and $L$ the circumference of the accelerator. See Appendix D for the derivation. Note that the slowly varying space charge potential $\tilde{V}_{n r, s c}$ does not depend on $s$, as Eq. (6) is an average over $s$.

\section{F. Incoherent amplitude-dependent detuning}

If we consider the case in which $\rho_{s}$ is small (zero), the canonical equations become

$$
\begin{gathered}
\tilde{a}_{\chi}^{\prime}=0, \\
\tilde{\varphi}_{\chi}^{\prime}=\frac{\partial \tilde{V}_{n r}}{\partial \tilde{a}_{\chi}}+t_{\chi} \frac{2 \pi \Delta_{r 0}}{L} .
\end{gathered}
$$

Integrating, we find $\tilde{a}_{\chi}=\tilde{a}_{\chi 0}$, and, returning back to the laboratory frame, we find $\hat{a}_{\chi}=\hat{a}_{\chi 0}$ and

$$
\hat{\varphi}_{\chi}(s)=\hat{\varphi}_{\chi 0}+\int_{0}^{s} \frac{\partial \tilde{V}_{n r}}{\partial \tilde{a}_{\chi}} d s^{\prime}
$$

In one turn, the quantities $\Delta \hat{\varphi}_{\chi}=\hat{\varphi}_{\chi}(L)-\hat{\varphi}_{\chi}(0)$ are simply the difference in phase advance between the perturbed phase advance and the phase advance of the unperturbed system. As $\hat{a}_{x}$ and $\hat{a}_{y}$ are constant, also the potential $\tilde{V}_{n r}$ is constant. Therefore, if no resonance acts on the particle, we find

$$
\Delta \hat{\varphi}_{\chi}=\frac{\partial \tilde{V}_{n r}\left(\hat{a}_{x}, \hat{a}_{y}\right)}{\partial \hat{a}_{\chi}} 2 \pi R
$$

which is an amplitude-dependent detuning, recall that $\hat{a}_{\chi} \rightarrow \tilde{a}_{\chi}$. If $\tilde{V}_{n r}$ is from space charge then in the limit case of $\hat{a}_{\chi} \rightarrow 0$, we retrieve the usual approximation for the incoherent space charge detuning. See Appendix E.

\section{G. Reduced system}

The canonical equations arising from the Hamiltonian Eq. (5) show the existence of the invariant $N_{y} \tilde{a}_{x}=N_{x} \tilde{a}_{y}+C$ for any slowly varying potential $\tilde{V}_{n r}$. This happens because the dynamics of particles is here dominated by the resonance. Therefore, once $C$ is fixed, one of the dynamical variables, the $\tilde{a}_{x}$ or the $\tilde{a}_{y}$, is redundant. We will keep the $\tilde{a}_{x}$ and call $\mathrm{a}=\tilde{a}_{x}$. In addition, by defining the variable $\Phi=N_{x} \tilde{\varphi}_{x}+N_{y} \tilde{\varphi}_{y}+\alpha$, the two canonical equations for $\tilde{\varphi}_{x}^{\prime}$ and $\tilde{\varphi}_{y}^{\prime}$ combine in the equation for $\Phi^{\prime}$. Therefore, the four canonical equations for the dynamical variables $\tilde{a}_{x}, \tilde{\varphi}_{x}, \tilde{a}_{y}$, and $\tilde{\varphi}_{y}$ can be reduced into two first-order differential equations in the new dynamical variables a and $\Phi$, defining a reduced system controlled by the Hamiltonian

$\mathrm{H}^{C}=\frac{4 \rho_{s}}{R} \mathrm{a}^{n_{x} / 2} \mathrm{a}_{y}^{n_{y} / 2} \cos [\Phi] N_{x}+\frac{\Delta_{r 0}}{R} \mathrm{a}+N_{x} \mathrm{~V}(\mathrm{a}, \mathrm{C})$,

with $\mathrm{V}(\mathrm{a}, \mathrm{C})=\tilde{V}_{n r}\left(\mathrm{a}, \mathrm{a}_{y}\right), \mathrm{C}=C$ (see Appendix F).

The use of Eq. (11) implies that the symbol $a_{y}$ is not a dynamical variable but rather a well-defined function of a via the relation $N_{y} \mathrm{a}=N_{x} \mathrm{a}_{y}+\mathrm{C}$, as $N_{x} \neq 0$ because our discussion is limited to coupled sum resonances. The domain of $a$ is now determined by the constraints $a_{y} \geq 0$ and $a \geq 0$.

\section{H. Stationary solutions}

The stationary solution of the canonical equations in the reduced system $C$,

$$
\mathbf{a}^{\prime}=-\frac{\partial \mathbf{H}^{C}}{\partial \Phi}, \quad \Phi^{\prime}=\frac{\partial \mathbf{H}^{C}}{\partial \mathbf{a}},
$$

allows finding the stationary solution of the canonical equations from the Hamiltonian Eq. (5) in a proper coordinate system, that is, a proper $t_{x}$, and vice versa establishing a relation between $\mathrm{C}$ and $t_{x}$ (see Appendix F). The stationary solution for the variables $\mathrm{a}(s)$ and $\Phi(s)$ or the variables $\tilde{a}_{\chi}(s)$ and $\tilde{\varphi}_{\chi}(s)$ is a fixed point, which in the physical coordinates $x, x^{\prime}, y, y^{\prime}$ has the topology of a "fixed line." The form of a fixed line is discussed in Appendix G.

Reference [16] discusses the fixed lines and their shape in the proximity of a third-order normal resonance in the case of no space charge and other sources of detuning. The stationary points for that resonance are shown in Fig. 2, with red for the stable and with blue for unstable 


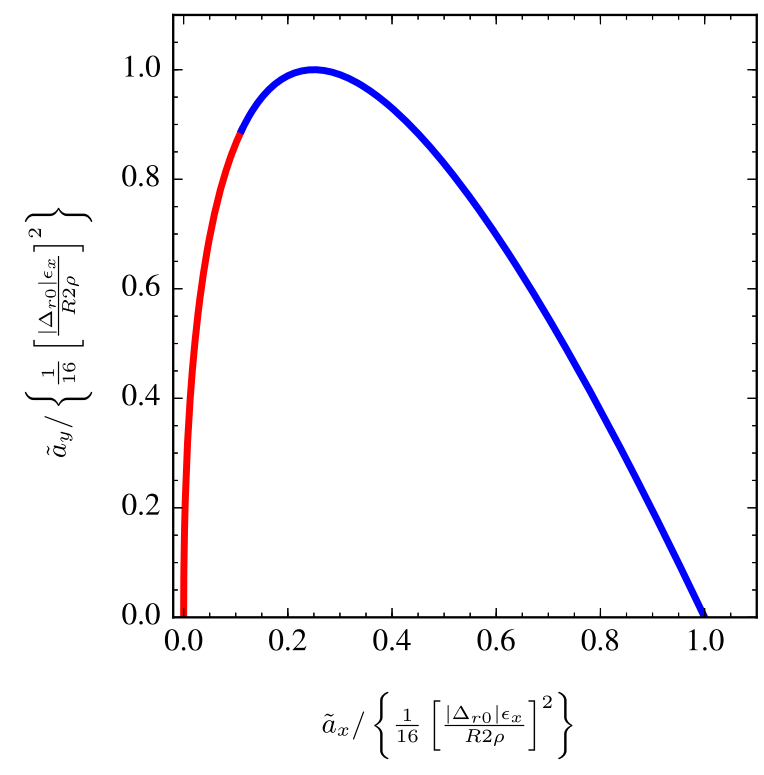

FIG. 2. Collection of all stationary points for the third-order normal resonance. In red are the stable and in blue the unstable solutions. In this picture for convenience we set $\rho=\rho_{n_{x}, n_{y}, j_{x}, j_{y}-m}$.

ones. The stability of the fixed points depends on the frequency of oscillations of neighbor particles. For imaginary frequencies, the fixed point becomes unstable. See Appendix F 1 for a discussion on the stability properties. Each of the points in Fig. 2 generates, in the physical coordinates, a fixed line [16] (see Appendix G). An extension of the results of Ref. [16] is presented in Appendix $\mathrm{H}$ for any order of the sum resonance including the stability properties.

\section{EFFECT OF THE INCOHERENT SPACE CHARGE ON FIXED LINES}

When the space charge is not negligible, the results of Fig. 2 are not valid, and the stationary points should be recomputed using the Hamiltonian Eq. (11). The stationary solutions of the canonical equations must now simultaneously satisfy the relations $\Phi=\pi q$ and

$$
\begin{gathered}
\frac{2 \rho_{s}}{R}\left[N_{x} \frac{n_{x}}{\mathrm{a}}+N_{y} \frac{n_{y}}{\mathrm{a}_{y}}\right] \mathrm{a}^{n_{x} / 2} \mathrm{a}_{y}^{n_{y} / 2}(-1)^{q} \\
+\frac{\Delta_{r 0}}{R}+N_{x} \frac{d}{d \mathrm{a}} \mathrm{V}(\mathrm{a}, \mathrm{C})=0 .
\end{gathered}
$$

The coefficient $q=0,1$ distinguishes between stable and unstable solutions for weak resonances. See Appendix F 1 for a discussion on the stability.

In Appendix I is shown that the potential $\mathrm{V}(\mathrm{a}, \mathrm{C})$ can be rescaled in the following form:

$$
\mathrm{V}(\mathrm{a}, \mathrm{C})=\frac{\mathcal{D}_{r, s c}}{R} \mathcal{V}_{\mathrm{sc}}(\mathrm{a}, \mathrm{C})
$$

with $\mathcal{D}_{r, s c}=N_{x} \Delta Q_{x}+N_{y} \Delta Q_{y}$ and $\Delta Q_{x}, \Delta Q_{y}$ the incoherent space charge tune shift in the center of the transverse distribution. The function $\mathcal{V}_{\mathrm{sc}}(\mathrm{a}, \mathrm{C})$ is a pure geometrical function which depends on the beam emittance ratio $\epsilon_{r}$, the resonance coefficients $N_{x}$ and $N_{y}$, and the type of beam distribution $F^{\prime}(t)$; for a Gaussian distribution, the function $\mathcal{V}_{\text {sc }}(\mathrm{a}, \mathrm{C})$ has the property $0 \leq N_{x} d \mathcal{V}_{\text {sc }}(\mathrm{a}, \mathrm{C}) / d \mathrm{a} \leq 1$, and, in the limit $\mathrm{a}=\mathrm{C}=0$, it yields $N_{x} d \mathcal{V}_{\text {sc }}(0,0) / d \mathrm{a}=1$. See Appendix I for a discussion on $\mathcal{V}_{\mathrm{sc}}$. Alternatively, in terms of amplitude-dependent detuning, we find

$\mathcal{D}_{r, s c} N_{x} \frac{d}{d \mathrm{a}} \mathcal{V}_{\mathrm{sc}}(\mathrm{a}, \mathrm{C})=N_{x} \Delta \mathrm{Q}_{x}(\mathrm{a}, \mathrm{C})+N_{y} \Delta \mathrm{Q}_{y}(\mathrm{a}, \mathrm{C})$

where

$$
\begin{aligned}
& \Delta \mathrm{Q}_{x}(\mathrm{a}, \mathrm{C})=\frac{\partial}{\partial \tilde{a}_{x}} \tilde{V}_{n r, s c}\left(\mathrm{a}, \frac{N_{y} \mathrm{a}-\mathrm{C}}{N_{x}}\right) R, \\
& \Delta \mathrm{Q}_{y}(\mathrm{a}, \mathrm{C})=\frac{\partial}{\partial \tilde{a}_{y}} \tilde{V}_{n r, s c}\left(\mathrm{a}, \frac{N_{y} \mathrm{a}-\mathrm{C}}{N_{x}}\right) R
\end{aligned}
$$

are the amplitude-dependent detuning. Note that Eqs. (16) and (17) mean to take $\tilde{V}_{n r, s c}\left(\tilde{a}_{x}, \tilde{a}_{y}\right)$, make the partial derivative, and then replace $\tilde{a}_{x} \rightarrow \mathrm{a}$ and $\tilde{a}_{y} \rightarrow \mathrm{a}_{y}$. Therefore, the equation of the stationary condition Eq. (13) reads

$$
\Delta_{r 0}+N_{x} \Delta \mathrm{Q}_{x}(\mathrm{a}, \mathrm{C})+N_{y} \Delta \mathrm{Q}_{y}(\mathrm{a}, \mathrm{C}) \propto \rho_{s}
$$

If the scaled driving term $\rho_{s}$ is small, i.e., for weak resonances, the solution is found at the amplitudes where the depressed single-particle tunes satisfy the resonance condition $N_{x} Q_{x}+N_{y} Q_{y}=m$, as intuitively expected.

This result is a direct consequence of the canonical equations of the slow variables, and it allows one to extend the concept of amplitude-dependent detuning to an "amplitude-dependent resonance detuning"

$$
\Delta_{r}(\mathrm{a}, \mathrm{C})=\Delta_{r 0}+N_{x} \mathcal{D}_{r, s c} \frac{d}{d \mathrm{a}} \mathcal{V}_{\mathrm{sc}}(\mathrm{a}, \mathrm{C})
$$

now defined for sum resonances of any order. The stationary solution, also known as the resonance, also known as the fixed line, is found when $\Delta_{r}(\mathrm{a}, \mathrm{C})=0$, and this formula shows how the distance from the resonance $\Delta_{r 0}$ and the resonant detuning $\mathcal{D}_{r, s c}$ affect the solution [as $\mathcal{V}_{\mathrm{sc}}(\mathrm{a}, \mathrm{C})$ is a purely geometrical function]. Note that the stationary solution $\mathrm{a}$ is also parametrized by $\mathrm{C}$, indicating that even in the presence of space charge there are infinite fixed lines with different properties of stability. 


\section{AN EXAMPLE OF APPLICATION}

In the following, the effect of space charge on the dynamics will be discussed, applying this theory to a machine experiment. It is considered a working example with $\Delta_{r 0}=0.056, \mathcal{D}_{r, s c}=-0.174$ corresponding to the settings, beam sizes, and space charge tune shifts of the CERN-PS measurements of Ref. [22]. With these settings, the parameters of incoherence are $\mathcal{I}_{x, l} \simeq \mathcal{I}_{y, l} \simeq 5$, somehow justifying the use of the frozen model. In all the next results, the beam distribution is Gaussian; hence, the generating function $F$ becomes $F(t)=-\exp (-t / 2)$.

The simulations presented next model the lattice as constant focusing for the purpose of speeding up the tracking computation. In fact, the tracking is performed by numerically computing the frozen space charge force from $V_{\mathrm{sc}}$ [Eq. (3)] via numerical integration. The fixed lines are instead computed solving numerically Eq. (13) using Eq. (14). For the specific example of the experimental settings of Ref. [22], the scaled driving term is $\rho_{s} \simeq 10^{-5}$.

In Fig. 3, we show the set of the stationary points obtained by solving Eq. (13). Figure 3 compared with Fig. 2 shows that the space charge created by the Gaussian distribution stabilized the otherwise unstable stationary points and dramatically changed the shape of the set in the neighborhood of $\tilde{a}_{x}=\tilde{a}_{y}=0$. Figure 2 also shows that stationary solutions scale proportionally to $\left|\Delta_{r 0}\right|^{2}$. However, for high-intensity beams, solving Eq. (13) we find that for $\Delta_{r 0} \rightarrow 0$ the amplitude of stationary points in Fig. 3 becomes larger and larger, whereas for $\Delta_{r 0} \rightarrow \mathcal{D}_{r, s c}$ it converges to zero. These features arise from the differences of the amplitude-dependent detuning created by space charge with respect to that created by magnets.

The theoretical approach presented allows the computation of the fixed lines for intense beams, for sum

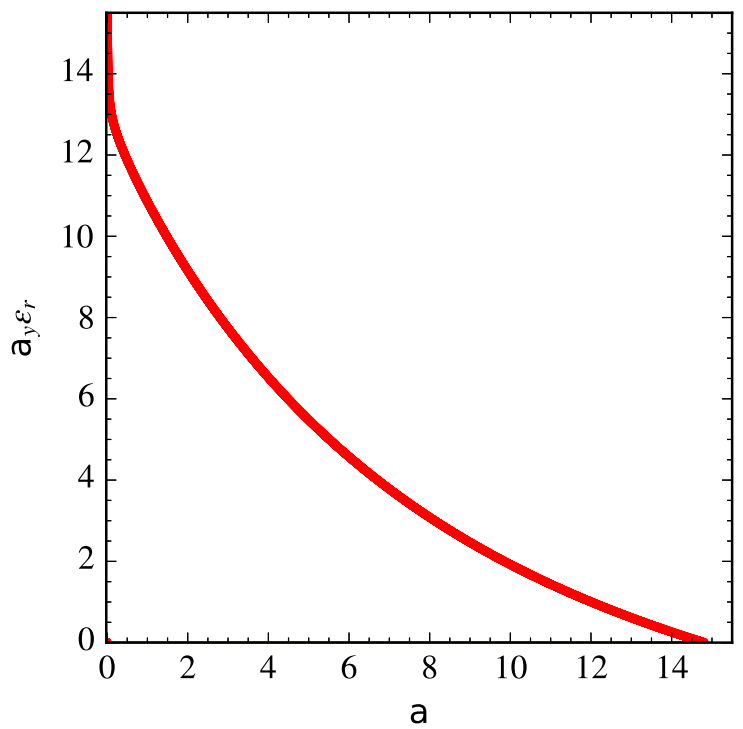

FIG. 3. Collection of all stationary points for the third-order normal resonance in a high-intensity coasting beam.

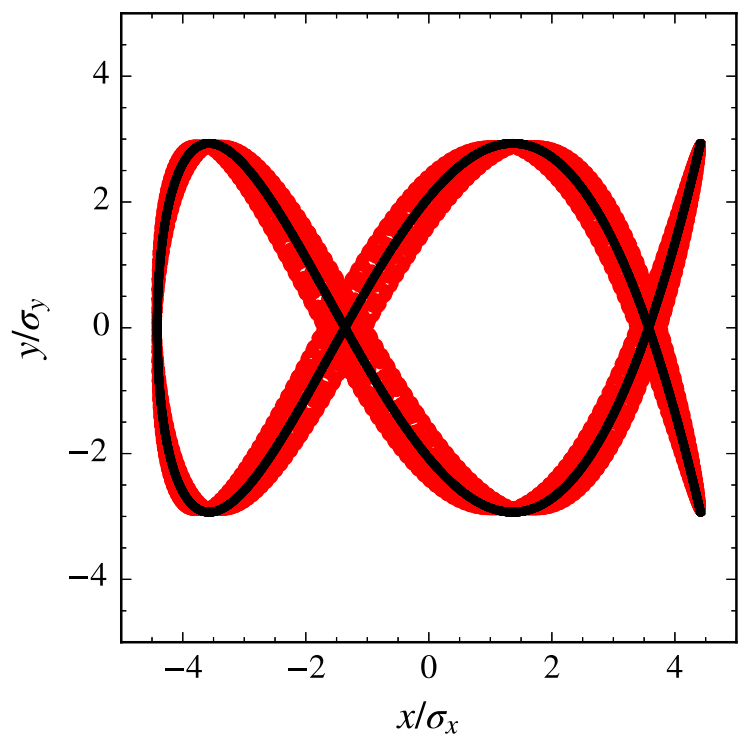

FIG. 4. Normalized projection of a $\mathrm{C}=20$ fixed line excited by the seventh-order resonance $5 Q_{x}+2 Q_{y}=m$ in the presence of space charge.

resonances of any order, and for space charge of arbitrary strength as long as in the incoherent regime. In Fig. 4, we show an example of a fixed line excited by the seventhorder normal resonance $5 Q_{x}+2 Q_{y}=m$ for the parameter $\mathrm{C}=20$. The red dots show the particle positions obtained via tracking, and the black dots the result from the theory. The resonance is properly driven by an ad hoc nonlinear element activated to the purpose of demonstration.

The Hamiltonian Eq. (11) can also be used to compute the amplitude of the tori embedding the fixed lines. This is shown in Fig. 5 for the example of the third-order normal resonance and $\mathrm{C}=-5$. The green orbits surround the

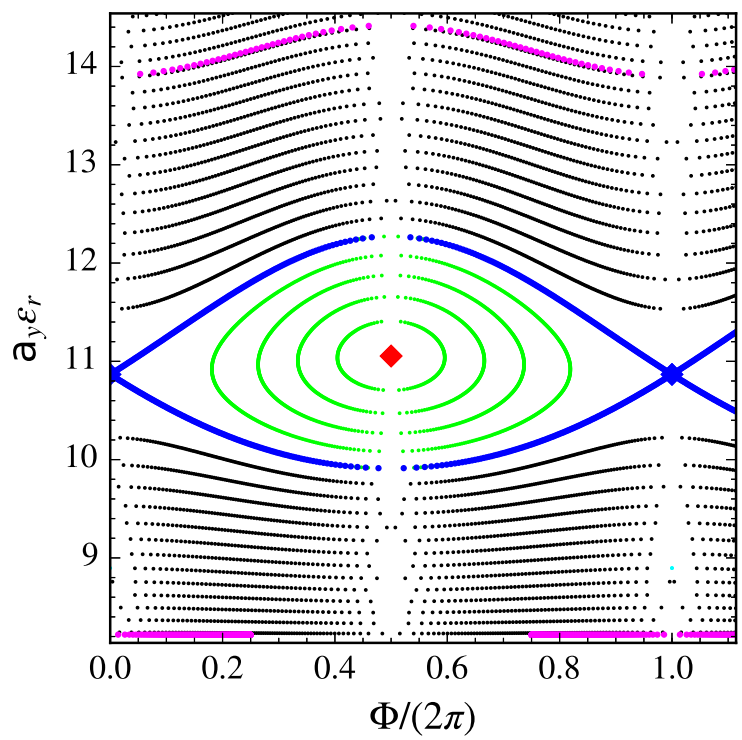

FIG. 5. Fixed line and tori for $\mathrm{C}=-5$ in a high-intensity coasting beam. 


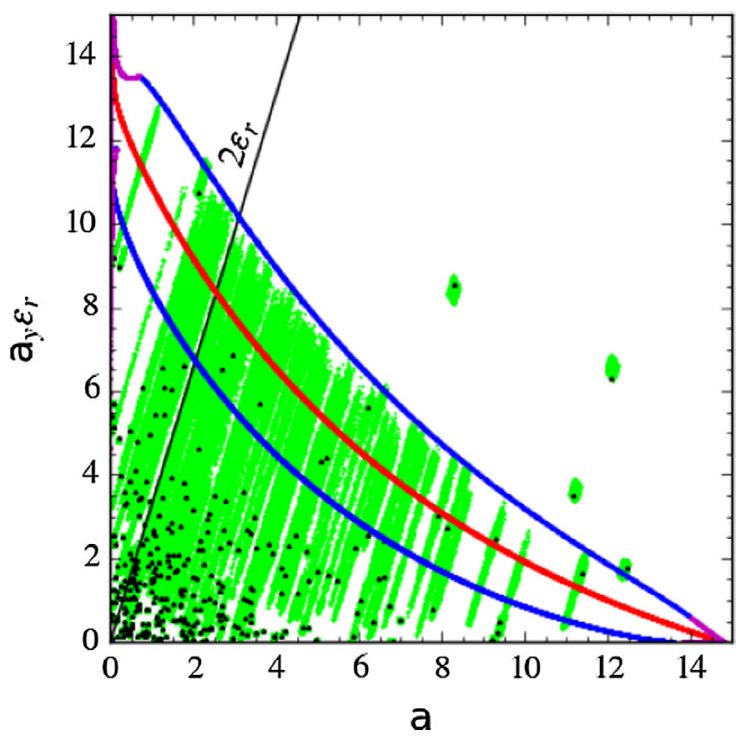

FIG. 6. Collection of all stationary points (red line) and outer and inner edges of the separatrix (blue line) in a high-intensity bunched beam. The green traces are the evolution of particles during storage.

stationary solution of the canonical equations, the red marker, and their topology is the analog of the phase space orbits in the islands for one-dimensional resonances. The red marker corresponds to a stationary fixed line. The blue curve is the separatrix, which overlaps the unstable fixed line (blue marker). Therefore, for every $\mathrm{C}$, a picture such as Fig. 5 allows finding the stationary point and the outer and inner extension of the separatrix. Collecting these points in a $\left(a, a_{y} \epsilon_{r}\right)$ diagram, we can extend the result of Fig. 3, adding two additional curves as shown in Fig. 6 (the two blue curves).

\section{A CHARACTERIZATION OF THE PERIODIC CROSSING OF FIXED LINES}

In a long bunched beam, particles are subject to an instantaneous space charge function of their longitudinal position $z / \sigma_{z}$ with respect to the bunch center. Here, $\sigma_{z}$ is the rms length of the bunch. In terms of resonance detuning, we typically find a local resonance detuning $\mathcal{D}_{r, s c}(z)=\mathcal{D}_{r, s c}(0) \exp \left(-0.5 z^{2} / \sigma_{z}^{2}\right)$. As the synchrotron motion pushes the bunch particles to oscillate around the bunch center, each of them will experience a modulation of $\mathcal{D}_{r, s c}(z)$ due to their motion $z(s)$. Hence, a bunch particle sees an instantaneous structure of fixed lines periodically spanning the transverse phase space as established in Ref. [22]. However, it is unclear among the infinite fixed lines which one will "cross" this bunch particle. To answer this question, a numerical study is carried out by taking 300 macroparticles in a linear bucket initialized as transverse Gaussian and longitudinal parabolic distribution. This bunch is tracked for $5 \times 10^{5}$ turns, and the dynamics is represented plotting $a, a_{y} \epsilon_{r}$, for each particle along the storage. In Fig. 6, the initial condition for each particle is a black marker, and the green paths are the value of $a, a_{y} \epsilon_{r}$ along the storage, which shows a diffusion due to the periodic crossing of the fixed lines. Surprisingly, it is found that particles hold their invariant, although the diffusive process caused by the repeated "scattering" and sometimes by "trapping" leading them up to the outer edge of the separatrix (the external blue curve). Particles not crossed by the fixed lines tori do not diffuse, as shown in Fig. 6 by the three particles located beyond the reach of the outer edge of the separatrix. Figure 6 immediately helps to interpret of Ref. [22] why in that experiment the beam distribution developed tails mainly in the vertical plane; it also helps to explain the phenomenology of the "adiabatic limit". In fact, in that investigation (Ref. [22]) test particles were set to small transverse amplitudes to a specific longitudinal location in a bunch with artificially small synchrotron tune (adiabatic limit). When trapped, these particles lock to fixed lines neighbor of the solid line of Fig. 6, hence are lead to "verge" towards the largest fixed-line which approximate the edge of the vertical halo.

\section{SUMMARY}

In this article, we have proposed to use the parameter $\mathcal{I}_{\chi, l}$, based on a plasma physics argument, to define the incoherent space charge regime and therefore to anticipate the goodness of space charge frozen models. We have presented the theory of the sum resonances with the frozen space charge. The theory confirms that the amplitudedependent resonance detuning $\Delta_{r}(\mathrm{a}, \mathrm{C})$ may be used for locating the fixed lines. We find that the theoretical predictions of fixed lines are in good agreement with particle tracking (Fig. 4). In the reduced system, the discussion of sum resonances with space charge becomes simple and allows easy identification of the set of stationary points and inner and outer edge of the separatrix (Fig. 6). These quantities define the key features for discussing the periodic crossing of fixed lines. Simulations also show the nonobvious result that particles, in good approximation, remain locked to the invariant $N_{y} \mathrm{a}=N_{x} \mathrm{a} y+\mathrm{C}$ during synchrotron oscillations in bunched beams. Therefore, a surprisingly simple method to predict the extension of the induced halo formation follows. The initial beam distribution should be represented in a $\left(\mathrm{a}, \mathrm{a}_{y} \epsilon_{r}\right)$ diagram, and, from each point, a line with the slope $\left(N_{y} / N_{x}\right) \epsilon_{r}$ is drawn until it intercepts the outer edge of the separatrix. These interception points will determine the outer boundary of the particle's diffusion.

The results presented in this study shed light on the complex phenomenon of the periodic crossing of sum resonances, although the theory presented includes only the first-order effect of the nonlinear errors. Further investigations to consolidate this approach will be part of future studies. 


\section{ACKNOWLEDGMENTS}

The author thanks M. Steck for the comments. This work was supported in part by the European Commission under the HORIZON2020 Integrating Activity project ARIES, Grant Agreement No. 730871.

\section{APPENDIX A: DEFINITION OF $\Delta Q_{\chi}$}

The use of Eq. (2) requires an operational definition of $\Delta Q_{\chi}$, with the convention $\chi=x, y$. Next, we consider a matched coasting beam with the accelerator quadrupolar structure having a general particle distribution

$$
n(x, y, s)=\frac{N}{\pi \sigma_{x}(s) \sigma_{y}(s)} F^{\prime}\left(\frac{x^{2}}{\sigma_{x}^{2}(s)}+\frac{y^{2}}{\sigma_{y}^{2}(s)}\right),
$$

where $N$ is the particle line density and $\sigma_{\chi}(s)$ are the rms beam sizes at location $s$. We assume here this distribution to be stationary; i.e., $F^{\prime}(t)$ does not change with the time ( $t$ is an integration variable, not the time). The function $F^{\prime}(t)$ in Eq. (A1) defines the type of beam distribution and must satisfy the conditions

$$
\int_{0}^{\infty} F^{\prime}(t) d t=1, \quad \int_{0}^{\infty} t F^{\prime}(t) d t=2 .
$$

The most common types of distribution are Gaussian with $F^{\prime}(t)=\frac{1}{2} \exp \left(-\frac{t}{2}\right)$, water bag with $F^{\prime}(t)=\frac{1}{3}\left(1-\frac{t}{6}\right)$, and $\mathrm{KV}$ with $F^{\prime}(t)=\frac{1}{4} \Theta\left(1-\frac{t}{4}\right), \Theta()$ being the Heaviside step function.

This particle beam in virtue of the rms equivalence [27] satisfies the envelope equations

$$
\sigma_{\chi}^{\prime \prime}+k_{\chi}(s) \sigma_{\chi}-\frac{K}{2\left(\sigma_{x}+\sigma_{y}\right)}-\frac{\epsilon_{\chi}^{2}}{\sigma_{\chi}^{3}}=0,
$$

with $K$ the beam perveance, $\epsilon_{\chi}$ the beam rms emittance, and $\sigma_{\chi}$ the rms envelope. The equation of the single particle very close to the beam center is

$$
\chi^{\prime \prime}+k_{\chi}(s) \chi-\frac{2 F^{\prime}(0) K}{\sigma_{\chi}\left(\sigma_{x}+\sigma_{y}\right)} \chi=0,
$$

and the corresponding equation of the beta functions (for this particle) including space charge is

$$
-\beta_{\chi} \alpha_{\chi}^{\prime}-\alpha_{\chi}^{2}+K_{\chi} \beta_{\chi}^{2}=1
$$

with $\alpha_{\chi}=-\beta_{\chi}^{\prime} / 2$ and

$$
K_{\chi}(s)=k_{\chi}(s)-\frac{2 F^{\prime}(0) K}{\sigma_{\chi}(s)\left[\sigma_{x}(s)+\sigma_{y}(s)\right]} .
$$

The depressed tune according to the Courant-Snyder definition is

$$
Q_{\chi}=R\left\langle\frac{1}{\beta_{\chi}}\right\rangle
$$

with \langle\rangle the average along the circumference. From Eq. (A2), we find the maximum incoherent space charge tune shift $\Delta Q_{\chi}=Q_{\chi}-Q_{\chi 0}$. This procedure allows computing $\Delta Q_{\chi}$ for an arbitrary accelerator structure and for an arbitrary intensity.

If the accelerator optics of an alternating gradient (AG) structure does not oscillate too much, in a good approximation it is

$$
\frac{Q_{\chi 0}^{2}}{R^{2}}-\frac{\left(Q_{\chi 0}+\Delta Q_{\chi}\right)^{2}}{R^{2}} \simeq 2 F^{\prime}(0) K \frac{1}{\bar{\sigma}_{\chi}\left(\bar{\sigma}_{x}+\bar{\sigma}_{y}\right)}
$$

with $R$ the accelerator average radius, $\Delta Q_{\chi}$ the maximum incoherent tune shift, and $\bar{\sigma}_{x}$ and $\bar{\sigma}_{y}$ the rms equivalent sizes in a constant focusing structure $\bar{\sigma}_{\chi}=$ $\left\{\left[R /\left(Q_{\chi 0}+\Delta Q_{\chi}\right)\right] \epsilon_{\chi}\right\}^{1 / 2}$. For a given accelerator lattice set to machine tunes $Q_{\chi 0}$, consider a beam with perveance $K$ and rms emittances $\epsilon_{x}$ and $\epsilon_{y}$; then solving Eq. (A3) means finding the maximum space charge incoherent tune shift $\Delta Q_{\chi}$. This expression predicts the maximum tune shift in a relatively good approximation in AG structures with beta oscillations up to $\sim 30 \%$. For small tune shifts, Eq. (A3) retrieves the usual expression

$$
\Delta Q_{\chi} \simeq-\frac{R^{2}}{Q_{\chi 0}} \frac{F^{\prime}(0) K}{\bar{\sigma}_{\chi}\left(\bar{\sigma}_{x}+\bar{\sigma}_{y}\right)} .
$$

\section{APPENDIX B: DERIVATION OF $\mathcal{I}_{\chi, l}$}

We discuss here the derivation of the ratio $\mathcal{I}_{\chi, l}$. The Debye length is defined as $\lambda_{D, \chi}=\tilde{v}_{\chi, \mathrm{th}} / \omega_{p}$, where $\tilde{v}_{\chi, \mathrm{th}}$ is the $\chi=x, y$ thermal component of the particle velocity and $\omega_{p}$ is the plasma frequency $\omega_{p}=\left[q^{2} n /\left(\epsilon_{0} \gamma^{3} m\right)\right]^{1 / 2}$, with $q$ the particle charge, $\epsilon_{0}$ the vacuum permittivity, $\gamma$ the Lorentz factor, and $m$ the particle mass; hence,

$$
\lambda_{D, \chi}=\left(\frac{\epsilon_{0} \gamma^{3} m \tilde{v}_{\chi, \mathrm{th}}^{2}}{q^{2} n}\right)^{1 / 2}
$$

At a given position $s$, the particle density depends on $(x, y)$ : We call the largest $n(x, y, s)$ by $n_{c}(s)$. The largest particle density yields the smaller Debye length, and for almost all beams the largest density is obtained at $x=y=0$; hence,

$$
n_{c}(s)=\frac{N}{\pi \sigma_{x}(s) \sigma_{y}(s)} F^{\prime}(0) .
$$

Therefore, at the location $s$ the smaller Debye length is

$$
\lambda_{D, \chi, \min }^{2}(s)=\frac{\pi \epsilon_{0} \gamma^{3} m \tilde{v}_{\chi, \mathrm{th}}^{2}(s) \sigma_{x}(s) \sigma_{y}(s)}{q^{2} N F^{\prime}(0)} .
$$


As the beam current is $I=q N v_{0}$, with $v_{0}$ the longitudinal particle velocity, and the beam perveance is defined as $K=$ $q I /\left(2 \pi \epsilon_{0} m \gamma^{3} \beta^{3} c^{3}\right)$ where $\beta=v_{0} / c$ and $c$ the speed of the light, we find

$$
\frac{\lambda_{D, \chi, \min }^{2}(s)}{\sigma_{x}(s) \sigma_{y}(s)}=\frac{1}{2 F^{\prime}(0) K} \frac{\tilde{v}_{\chi, \mathrm{th}}^{2}(s)}{v_{0}^{2}} .
$$

For a beam matched with the space charge depressed linear optics, the beam phase space is linearly correlated in $x, x^{\prime}$; hence, the thermal rms velocity $\tilde{v}_{\chi \text {,th }}$ satisfies the relation

$$
\epsilon_{\chi}=\sigma_{\chi}(s) \frac{\tilde{v}_{\chi, \mathrm{th}}(s)}{v_{0}}
$$

where $\epsilon_{\chi}$ is the beam rms emittance. Therefore, we find the following expression:

$$
\frac{\lambda_{D, \chi, \min }^{2}(s)}{\sigma_{x}(s) \sigma_{y}(s)}=\frac{1}{2 F^{\prime}(0) K} \frac{\epsilon_{\chi}^{2}}{\sigma_{\chi}^{2}(s)} .
$$

To make this formula more practical, we estimate the perveance $K$ via the incoherent space charge tune shift $\Delta Q_{\chi}$ and the matched beam sizes.

Given an AG structure of which are known $R, Q_{\chi 0}$, and assuming this structure transporting an intense beam of emittance $\epsilon_{\chi}$ creating a space charge tune shift $\Delta Q_{\chi}$, then using Eq. (A3) allows retrieving $2 F^{\prime}(0) K$, i.e. the beam perveance, as well as the rms matched beam sizes $\bar{\sigma}_{\chi}$. These quantities can be replaced in Eq. (B2), and using the approximation $\left\langle\sigma_{\bar{\chi}} / \sigma_{\chi}^{3}\right\rangle \simeq\left\langle\sigma_{\bar{\chi}}\right\rangle /\left\langle\sigma_{\chi}\right\rangle^{3} \simeq \bar{\sigma}_{\bar{\chi}} / \bar{\sigma}_{\chi}^{3}$ which holds for smooth optics we find

$$
\mathcal{I}_{\chi} \simeq \sqrt{\frac{\bar{\sigma}_{\bar{\chi}}}{\bar{\sigma}_{x}+\bar{\sigma}_{y}}} \frac{1+\frac{\Delta Q_{\chi}}{Q_{\chi 0}}}{\sqrt{1-\left(1+\frac{\Delta Q_{\chi}}{Q_{\chi 0}}\right)^{2}}} \Lambda_{\chi},
$$

where $\bar{\chi}$ is the complement of $\chi$ and $\Lambda_{\chi}=\sqrt{\epsilon_{\chi}^{2} R^{2} /\left(\bar{\sigma}_{\chi}^{4} Q_{\chi}^{2}\right)}$. In this expression, the term $\epsilon_{\chi}^{2} / \bar{\sigma}_{\chi}^{4}$ can be evaluated from an rms KV equivalent beam having the same perveance and characterized by the incoherent depressed tune $Q_{k v, \chi}$; hence, $\Lambda_{\chi} \simeq Q_{k v, \chi} / Q_{\chi}$. Note that this quantity is always larger than 1 , as the depressed tune of any beam distribution with a peak density at the center of the beam is smaller than the one of its rms equivalent $\mathrm{KV}$ beam. Therefore, we reach the result

$$
\mathcal{I}_{\chi} \gtrsim \mathcal{I}_{\chi, l}=\sqrt{\frac{\bar{\sigma}_{\bar{\chi}}}{\bar{\sigma}_{x}+\bar{\sigma}_{y}}} \frac{1+\frac{\Delta Q_{\chi}}{Q_{\chi 0}}}{\sqrt{1-\left(1+\frac{\Delta Q_{\chi}}{Q_{\chi 0}}\right)^{2}}}
$$

with $\mathcal{I}_{\chi, l}$ the lower limit of $\mathcal{I}_{\chi}$. Equality is valid for a $\mathrm{KV}$ distribution.

\section{APPENDIX C: THE FORM OF $\hat{H}_{1}$}

The perturbation Hamiltonian $H_{1}$ is a function only of the space coordinates, which in a general form we write as

$$
H_{1}=\sum_{n_{x}, n_{y}} c_{n_{x}, n_{y}}(s) x^{n_{x}} y^{n_{y}}
$$

with the coefficient $c_{n_{x}, n_{y}}(s)$ depending on the potential created by the distribution of the nonlinear errors around the ring or on the space charge potential. The units of $c_{n_{x}, n_{y}}(s)$ come from units of $H_{1}$ and $x, y:\left[c_{x, n_{y}}\right] \equiv\left[m^{-n_{x}-n_{y}}\right]$. In scaled coordinates, the perturbation Hamiltonian $\hat{H}_{1}$ reads

$$
\hat{H}_{1}=\sum_{n_{x}, n_{y}} c_{n_{x}, n_{y}}(s) \epsilon_{x}^{\left(n_{x}+n_{y}\right) / 2} \hat{x}^{n_{x}} \hat{y}^{n_{y}}
$$

Substituting the modified Courant-Snyder form, we find

$$
\begin{aligned}
\hat{H}_{1}= & \sum_{n_{x}, n_{y}} c_{n_{x}, n_{y}} \sigma_{x}^{n_{x}} \sigma_{y}^{n_{y}} \epsilon_{r}^{n_{y} / 2} \\
& \times \cos ^{n_{x}}\left(\psi_{x}+\hat{\varphi}_{x}\right) \cos ^{n_{y}}\left(\psi_{y}+\hat{\varphi}_{y}\right) \hat{a}_{x}^{n_{x} / 2} \hat{a}_{y}^{n_{y} / 2},
\end{aligned}
$$

where we call $\epsilon_{r}=\epsilon_{x} / \epsilon_{y}$ the ratio of the transverse beam emittances. In order to simplify the notation, we omit the explicit $s$ dependence on all variables with the understanding that only $\epsilon_{r}$ and $\epsilon_{\chi}$ are constant. Note that in Eq. (C1) the beam is assumed to be matched with the optics; hence, $\sigma_{\chi}^{2}=\beta_{\chi} \epsilon_{\chi}$. Equation (C1) is a series formed by terms which are periodic in $s$ with period $L$, the machine length. Next, we can Fourier expand these terms with the periodicity of the circular accelerator, namely, expand in harmonics $e^{-i(2 \pi m / L) s}$. Therefore, we can recast Eq. (C1) as follows:

$$
\begin{aligned}
\hat{H}_{1}= & \sum_{n_{x}, n_{y}} \sum_{m=-\infty}^{\infty} \sum_{j_{x}=0}^{n_{x}} \sum_{j_{y}=0}^{n_{y}} \rho_{n_{x}, n_{y}, j_{x}, j_{y}, m} \hat{a}_{x}^{n_{x} / 2} \hat{a}_{y}^{n_{y} / 2} \\
& \times \cos \left[\Psi_{x}\left(2 j_{x}-n_{x}\right)+\Psi_{y}\left(2 j_{y}-n_{y}\right)\right. \\
& \left.+\frac{2 \pi m}{L} s+\alpha_{n_{x}, n_{y}, j_{x}, j_{y}, m}\right]
\end{aligned}
$$

with $\Psi_{\chi}=\frac{2 \pi Q_{\chi 0}}{L} s+\hat{\varphi}_{\chi}$. The coefficients $\rho_{n_{x}, n_{y}, j_{x}, j_{y}, m} \geq 0$ and $\alpha_{n_{x}, n_{y}, j_{x}, j_{y}, m}$ of the Fourier expansion are obtained from

$$
\begin{aligned}
f_{n_{x}, n_{y}, j_{x}, j_{y}, m}= & \frac{1}{L} \frac{1}{2^{n_{x}+n_{y}}}\left(\begin{array}{c}
n_{x} \\
j_{x}
\end{array}\right)\left(\begin{array}{c}
n_{y} \\
j_{y}
\end{array}\right) \\
& \times \int_{0}^{L} c_{n_{x}, n_{y}} \sigma_{x}^{n_{x}} \sigma_{y}^{n_{y}} \epsilon_{r}^{n_{y} / 2} \\
& \times e^{i\left[\mathcal{D}_{x}\left(2 j_{x}-n_{x}\right)+\mathcal{D}_{y}\left(2 j_{y}-n_{y}\right)-(2 \pi m / L) s\right]} d s,
\end{aligned}
$$

where $\mathcal{D}_{\chi}=\psi_{\chi}-\frac{2 \pi Q_{\chi 0}}{L} s$, and 


$$
f_{n_{x}, n_{y}, j_{x}, j_{y}, m}=\rho_{n_{x}, n_{y}, j_{x}, j_{y}, m} e^{i \alpha_{n_{x}, n_{y}, j_{x}, j_{y}, m}} .
$$

The coefficients $\rho_{n_{x}, n_{y}, j_{x}, j_{y}, m}$ and $\alpha_{n_{x}, n_{y}, j_{x}, j_{y}, m}$ incorporate the optical properties as well as the properties of the nonlinear potential from nonlinear magnets and space charge. The meaning of these coefficients is explained in Appendix D. In the next subsections, we show the explicit form of $f_{n_{x}, n_{y}, j_{x}, j_{y}, m}$ as created by space charge or magnetic components. These coefficients are essential to activate resonances and, hence, are related to the resonance driving terms.

\section{Space charge coefficients}

We derive in this section the space charge coefficients. The expression of the space charge potential Eq. (3) is conveniently expanded in the following series:

$$
V_{\mathrm{sc}}=-\frac{K}{2} \sum_{n_{x}+n_{y}>0} F_{\left(n_{x}+n_{y}\right)} \frac{1}{n_{x} ! n_{y} !} I_{2 n_{x}, 2 n_{y}} x^{2 n_{x}} y^{2 n_{y}}
$$

with $F_{(n)}=d F^{n} /\left.d t^{n}\right|_{0}$, and

$$
I_{n_{x}, n_{y}}=\int_{0}^{\infty} \frac{1}{\left(\sigma_{x}^{2}+t\right)^{\left(n_{x}+1\right) / 2}\left(\sigma_{y}^{2}+t\right)^{\left(n_{y}+1\right) / 2}} d t
$$

a coefficient $s$ dependent. Hence,

$$
c_{2 n_{x}, 2 n_{y}}=-\frac{K}{2} F_{\left(n_{x}+n_{y}\right)} \frac{1}{n_{x} ! n_{y} !} I_{2 n_{x}, 2 n_{y}},
$$

which substituted in Eq. (C3) yields $\rho_{2 n_{x}, 2 n_{y}, j_{x}, j_{y}, m}$ and $\alpha_{2 n_{x}, 2 n_{y}, j_{x}, j_{y}, m}$. We find

$$
\begin{aligned}
& f_{2 n_{x}, 2 n_{y}, j_{x}, j_{y}, m} \\
& =-\frac{K}{L} \frac{F_{\left(n_{x}+n_{y}\right)}}{2^{2\left(n_{x}+n_{y}\right)+1}}\left(\begin{array}{c}
2 n_{x} \\
j_{x}
\end{array}\right)\left(\begin{array}{c}
2 n_{y} \\
j_{y}
\end{array}\right) \frac{1}{n_{x} ! n_{y} !} \epsilon_{r}^{n_{y}} \\
& \quad \times \int_{0}^{L} \mathcal{I}_{2 n_{x}, 2 n_{y}} e^{i\left[\mathcal{D}_{x}\left(2 j_{x}-2 n_{x}\right)+\mathcal{D}_{y}\left(2 j_{y}-2 n_{y}\right)-(2 \pi m / L) s\right]} d s,
\end{aligned}
$$

where $\mathcal{I}_{2 n_{x}, 2 n_{y}}=I_{2 n_{x}, 2 n_{y}} \sigma_{x}^{2 n_{x}} \sigma_{y}^{2 n_{y}}$ is an $s$-dependent dimensionless function, which first-order terms are easily found to be

$$
\mathcal{I}_{2,0}=\frac{2 \sigma_{x}}{\sigma_{x}+\sigma_{y}}, \quad \mathcal{I}_{0,2}=\frac{2 \sigma_{y}}{\sigma_{x}+\sigma_{y}}
$$

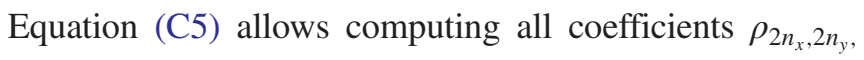
$j_{x}, j_{y}, m$ and $\alpha_{2 n_{x}, 2 n_{y}, j_{x}, j_{y}, m}$ which are necessary for including the effect of the space charge potential in the dynamics. For the particular case of a Gaussian distribution, $F_{(n)}=-(-1 / 2)^{n}$.

\section{Nonlinear magnet coefficients}

The potential for the magnetic field is

$$
V_{m}=\sum_{n=0}^{\infty} \operatorname{Re}\left[\left(k_{n}+i j_{n}\right) \frac{(x+i y)^{n+1}}{(n+1) !}\right],
$$

and the units are $\left[V_{m}\right] \equiv[1]$ and $\left[k_{n}\right] \equiv\left[j_{n}\right] \equiv\left[m^{-n-1}\right]$. The normal and skew components are $s$ dependent; however, the nonlinear components are usually localized and, hence, are approximated as

$$
k_{n}+i j_{n}=\sum_{i=1}^{N_{n}} \delta\left(s-s_{n, i}\right)\left(K_{n, i}+i J_{n, i}\right)
$$

with $K_{n, i}, J_{n, i}$ the integrated strength of the $i$ th nonlinear error located at the position $s_{n, i} \bmod L$, and $N_{n}$ the number of nonlinear errors around the ring. The coefficients $c_{n_{x}, n_{y}}$ are found from Eq. (C6). We find that for normal components

$$
c_{n_{x}, n_{y}}(s)=\sum_{i=1}^{N_{n}} K_{n, i} \delta\left(s-s_{n, i}\right) \frac{\cos \left(n_{y} \pi / 2\right)}{(n+1) !}\left(\begin{array}{c}
n+1 \\
n_{x}
\end{array}\right),
$$

where $n=n_{x}+n_{y}-1$, while for skew components

$$
c_{n_{x}, n_{y}}(s)=\sum_{i=1}^{N_{n}} J_{n, i} \delta\left(s-s_{n, i}\right) \frac{\cos \left[\left(n_{y}+1\right) \pi / 2\right]}{(n+1) !}\left(\begin{array}{c}
n+1 \\
n_{x}
\end{array}\right) .
$$

Therefore, replacing in Eq. (C3) we find

$$
f_{n_{x}, n_{y}, j_{x}, j_{y}, m}=\frac{1}{L} \frac{\cos \left[n_{y} \pi / 2\right]}{2^{n_{x}+n_{y}} n_{x} ! n_{y} !}\left(\begin{array}{c}
n_{x} \\
j_{x}
\end{array}\right)\left(\begin{array}{c}
n_{y} \\
j_{y}
\end{array}\right) \sum_{i=1}^{N_{n}} K_{n, i} \sigma_{x i}^{n_{x}} \sigma_{y i}^{n_{y}} \epsilon_{r}^{n_{y} / 2} e^{i\left[\mathcal{D}_{x i}\left(2 j_{x}-n_{x}\right)+\mathcal{D}_{y i}\left(2 j_{y}-n_{y}\right)-(2 \pi m / L) s_{n, i}\right]}
$$

for the normal components and

$$
f_{n_{x}, n_{y}, j_{x}, j_{y}, m}=-\frac{1}{L} \frac{\sin \left[n_{y} \pi / 2\right]}{2^{n_{x}+n_{y}} n_{x} ! n_{y} !}\left(\begin{array}{c}
n_{x} \\
j_{x}
\end{array}\right)\left(\begin{array}{c}
n_{y} \\
j_{y}
\end{array}\right) \sum_{i=1}^{N_{n}} J_{n, i} \sigma_{x i}^{n_{x}} \sigma_{y i}^{n_{y}} \epsilon_{r}^{n_{y} / 2} e^{i\left[\mathcal{D}_{x i}\left(2 j_{x}-n_{x}\right)+\mathcal{D}_{y i}\left(2 j_{y}-n_{y}\right)-(2 \pi m / L) s_{n, i}\right]}
$$


for the skew components. The quantities $\sigma_{x i}, \sigma_{y i}, \mathcal{D}_{x i}$, and $\mathcal{D}_{y i}$ are the periodic $s$-dependent functions $\sigma_{x}, \sigma_{y}, \mathcal{D}_{x}$, and $\mathcal{D}_{y}$, respectively, computed at $s=s_{n, i} \bmod L$. Equations (C7) and (C8) allow computing the coefficients $\rho_{n_{x}, n_{y}, j_{x}, j_{y}, m}$ and $\alpha_{n_{x}, n_{y}, j_{x}, j_{y}, m}$ for any nonlinear error distributions.

\section{APPENDIX D: THE SLOWLY VARYING HAMILTONIAN}

Equation (C2) shows that $\hat{H}_{1}$ is a series of oscillating terms. The frequency of the term with coefficient $\rho_{n_{x}, n_{y}, j_{x}, j_{y}, m}$ is

$$
\frac{2 \pi}{L}\left[Q_{x 0}\left(2 j_{x}-n_{x}\right)+Q_{y 0}\left(2 j_{y}-n_{y}\right)+m\right] s .
$$

We now consider the following approximations. (1) Terms of the series oscillating more rapidly do not play a significant role. The idea invoked here is that rapidly oscillating terms average out so that the slower oscillating terms dominate the dynamics. (2) Terms with coefficients $\rho_{n_{x}, n_{y}, j_{x}, j_{y}, m}$ smaller than other terms of the expansion can be neglected.

Therefore, if for a certain set of indexes $n_{x}, n_{y}, j_{x}, j_{y}, m$ we have $\rho_{n_{x}, n_{y}, j_{x}, j_{y}, m}>0$ and we set the tunes $Q_{x 0}, Q_{y 0}$ such that

$$
\left(2 j_{x}-n_{x}\right) Q_{x 0}+\left(2 j_{y}-n_{y}\right) Q_{y 0}+m \simeq 0,
$$

then we force the term with indexes $n_{x}, n_{y}, j_{x}, j_{y}, m$ to strongly influence the particle dynamics, which becomes a resonant dynamics. Setting $N_{x}=2 j_{x}-n_{x}$ and $N_{y}=2 j_{y}-n_{y}$, we define the distance from the resonance as

$$
\Delta_{r 0}=N_{x} Q_{x 0}+N_{y} Q_{y 0}-m .
$$

Therefore, the Hamiltonian has now a dominant slowly varying component, which defines a "resonant slowly varying Hamiltonian"

$\hat{H}_{r s}=\frac{4 \rho_{s}}{R} \hat{a}_{x}^{n_{x} / 2} \hat{a}_{y}^{n_{y} / 2} \cos \left[\frac{2 \pi \Delta_{r 0}}{L} s+\hat{\varphi}_{x} N_{x}+\hat{\varphi}_{y} N_{y}+\alpha\right]$.

In this formula, we have called for convenience of notation $\rho_{s}=\rho_{n_{x}, n_{y}, j_{x}, j_{y},-m} R / \epsilon_{x}$ and $\alpha=\alpha_{n_{x}, n_{y}, j_{x}, j_{y},-m}$. Note that the definition of $\hat{H}_{r s}$ in Eq. (D2) incorporates the term $2 / \epsilon_{x}$ present in the canonical equation (4). The extra factor of 2 arises because in expansion Eq. (C2) there are two identical terms. The coefficient $\rho_{s}$ is the scaled driving term.

We observe that Eq. (D2) is not the only slowly oscillating term of Eq. (C2); there are also other sets of indexes which generate slowly varying terms. This happens for all the terms with indexes $n_{x}$ and $n_{y}$ even, which also have $2 j_{x}=n_{x}, 2 j_{y}=n_{y}$, and $m=0$. In this case, Eq. (D1) is exactly zero. As these terms are $s$ independent regardless of the setting of $Q_{x 0}$ and $Q_{y 0}$, we refer to these term as "nonresonant." We call for convenience the sum of all nonresonant terms as $\hat{V}_{n r}$, which reads

$$
\hat{V}_{n r}=\sum_{n_{x}+n_{y}>0} \frac{2}{\epsilon_{x}} \rho_{2 n_{x}, 2 n_{y}, n_{x}, n_{y}, 0} \hat{a}_{x}^{n_{x}} \hat{a}_{y}^{n_{y}} \cos \left[\alpha_{\left.2 n_{x}, 2 n_{y}, n_{x}, n_{y}, 0\right]}\right] .
$$

For these particular indexes, we find that Eq. (C3) yields

$$
\begin{aligned}
f_{2 n_{x}, 2 n_{y}, n_{x}, n_{y}, 0}= & \frac{1}{L} \frac{1}{2^{2\left(n_{x}+n_{y}\right)}}\left(\begin{array}{c}
2 n_{x} \\
n_{x}
\end{array}\right)\left(\begin{array}{c}
2 n_{y} \\
n_{y}
\end{array}\right) \\
& \times \int_{0}^{L} c_{2 n_{x}, 2 n_{y}} \sigma_{x}^{2 n_{x}} \sigma_{y}^{2 n_{y}} \epsilon_{r}^{n_{y}} d s,
\end{aligned}
$$

and as $c_{2 n_{x}, 2 n_{y}}$ is real it follows that $f_{2 n_{x}, 2 n_{y}, n_{x}, n_{y}, 0}$ is real as well. Therefore,

$$
f_{2 n_{x}, 2 n_{y}, n_{x}, n_{y}, 0}=\rho_{2 n_{x}, 2 n_{y}, n_{x}, n_{y}, 0} \cos \left[\alpha_{2 n_{x}, 2 n_{y}, n_{x}, n_{y}, 0}\right],
$$

and we conclude that the more general form of the nonresonant slowly varying potential is

$$
\hat{V}_{n r}=\sum_{n_{x}+n_{y}>0} \frac{2}{\epsilon_{x}} f_{2 n_{x}, 2 n_{y}, n_{x}, n_{y}, 0} \hat{a}_{x}^{n_{x}} \hat{a}_{y}^{n_{y}} .
$$

In conclusion, the dynamics is dominated by the slowly varying perturbing Hamiltonian $\hat{H}_{1 s}=\hat{H}_{r s}+\hat{V}_{n r}$.

\section{Nonresonant slowly varying potential from space charge}

These special types of slowly varying terms are always generated by space charge, as the potential expansion has the terms $\rho_{2 n_{x}, 2 n_{y}, n_{x}, n_{y}, 0}$ different than zero. Replacing Eq. (C5) into Eq. (D4), we compute $\hat{V}_{n r}$ for the space charge. By making use of the identity

$$
2^{2 n} \frac{1}{2 \pi} \int_{0}^{2 \pi} \cos ^{2 n}(t) d t=\left(\begin{array}{c}
2 n \\
n
\end{array}\right)
$$

we obtain

$$
\begin{aligned}
\hat{V}_{n r, s c}= & \left\langle-\frac{K}{\epsilon_{x}} \frac{1}{2 \pi} \int_{0}^{2 \pi} d x \frac{1}{2 \pi} \int_{0}^{2 \pi} d y\right. \\
& \times \sum_{n_{x}+n_{y}>0} \frac{F_{\left(n_{x}+n_{y}\right)}}{n_{y} ! n_{x} !}\left[\cos ^{2}(x) \hat{a}_{x}\right]^{n_{x}}\left[\cos ^{2}(y) \epsilon_{r} \hat{a}_{y}\right]^{n_{y}} \\
& \left.\times \mathcal{I}_{2 n_{x}, 2 n_{y}}\right\rangle
\end{aligned}
$$


where we use the symbol \langle\rangle as the average over the machine circumference and $F_{(n)}=d^{n} F /\left.d t^{n}\right|_{0}$. Because of the special character of space charge where the forces in a 2D beam drops as $1 / r$, it follows that an approximation of the previous integral is not simple. The larger $\hat{a}_{x}$ and $\hat{a}_{y}$, the more terms of the expansion have to be included. Therefore, we are forced to compute the full function by summing the series. Recalling that $\mathcal{I}_{2 n_{x}, 2 n_{y}}=$ $I_{2 n_{x}, 2 n_{y}} \sigma_{x}^{2 n_{x}} \sigma_{y}^{2 n_{y}}$ and Eq. (C4), we find

$$
\begin{aligned}
\hat{V}_{n r, s c}\left(\hat{a}_{x}, \hat{a}_{y}\right)= & \frac{1}{2 \pi} \int_{0}^{2 \pi} d x \frac{1}{2 \pi} \int_{0}^{2 \pi} d y \\
& \times\left\langle\frac{2}{\epsilon_{x}} V_{\mathrm{sc}}\left[\cos (x) \sigma_{x} \sqrt{\hat{a}_{x}}, \cos (y) \sigma_{y} \sqrt{\epsilon_{r} \hat{a}_{y}}, s\right]\right\rangle .
\end{aligned}
$$

For a Gaussian distribution, this form can be rewritten in terms of modified Bessel functions $I_{0}(t)$ as

$$
\begin{aligned}
& \hat{V}_{n r, s c}\left(\hat{a}_{x}, \hat{a}_{y}\right) \\
& =-\frac{K}{\epsilon_{x}}\left\langle\int _ { 0 } ^ { \infty } d t \left[ 1-I_{0}\left(\frac{\sigma_{x}^{2}}{4\left(\sigma_{x}^{2}+t\right)} \hat{a}_{x}\right)\right.\right. \\
& \quad \times I_{0}\left(\frac{\sigma_{y}^{2}}{4\left(\sigma_{y}^{2}+t\right)} \epsilon_{r} \hat{a}_{y}\right) \exp \left[-\frac{\sigma_{x}^{2}}{4\left(\sigma_{x}^{2}+t\right)} \hat{a}_{x}-\frac{\sigma_{y}^{2}}{4\left(\sigma_{y}^{2}+t\right)} \epsilon_{r} \hat{a}_{y}\right] \\
& \left.\quad \times \frac{1}{\left(\sigma_{x}^{2}+t\right)^{1 / 2}\left(\sigma_{y}^{2}+t\right)^{1 / 2}}\right\rangle .
\end{aligned}
$$

This expression allows for a more efficient numerical computation of $\hat{V}_{n r, s c}$.

\section{Nonresonant slowly varying potential from nonlinear magnet components}

The nonresonant terms from magnets are computed in the same way, and we find that only a normal component may create nonresonant terms. With straightforward algebra, we find

$$
\begin{aligned}
\hat{V}_{n r, m}= & \sum_{n_{x}+n_{y}>0} \frac{2}{\epsilon_{x}} \frac{1}{L} \frac{\cos \left[n_{y} \pi\right]}{2^{2 n_{x}+2 n_{y}}} \frac{1}{\left(n_{x} !\right)^{2}\left(n_{y} !\right)^{2}} \\
& \times \sum_{i=1}^{N_{n}} K_{n, i} \sigma_{x i}^{2 n_{x}} \sigma_{y i}^{2 n_{y}} \epsilon_{r}^{n_{y}} \hat{a}_{x}^{n_{x}} \hat{a}_{y}^{n_{y}}
\end{aligned}
$$

where now $n=2 n_{x}+2 n_{y}-1$. Only the magnet components of strength $K_{n, i}$ with $n+1$ even will create a slowly varying potential.

\section{APPENDIX E: INCOHERENT SPACE CHARGE TUNE SHIFT}

The incoherent space charge detuning is obtained from the amplitude-dependent detuning when we consider a particle with a small transverse amplitude, hence, for $\tilde{a}_{x} \rightarrow 0, \tilde{a}_{y} \rightarrow 0$. The nonresonant potential Eq. (D5) is conveniently rearranged as

$$
\begin{aligned}
\tilde{V}_{n r, s c}= & -\sum_{n_{x}+n_{y}>0} \frac{K}{\epsilon_{x}} \frac{1}{2^{2\left(n_{x}+n_{y}\right)}}\left(\begin{array}{c}
2 n_{x} \\
n_{x}
\end{array}\right)\left(\begin{array}{c}
2 n_{y} \\
n_{y}
\end{array}\right) \frac{F_{\left(n_{x}+n_{y}\right)}}{n_{y} ! n_{x} !} \\
& \times\left\langle\mathcal{I}_{2 n_{x}, 2 n_{y}}\right) \tilde{a}_{x}^{n_{x}} \tilde{a}_{y}^{n_{y}} \epsilon_{r}^{n_{y}},
\end{aligned}
$$

and for small $\tilde{a}_{x}$ and $\tilde{a}_{y}$ it reads

$$
\tilde{V}_{n r, s c}=-\frac{K}{2 \epsilon_{x}} F_{(1)}\left\langle\mathcal{I}_{2,0}\right\rangle \tilde{a}_{x}-\frac{K}{2 \epsilon_{x}} F_{(1)}\left\langle\mathcal{I}_{0,2}\right\rangle \tilde{a}_{y} \epsilon_{r}-\cdots
$$

Therefore,

$$
\begin{gathered}
\frac{\partial \tilde{V}_{n r, s c}}{\partial \tilde{a}_{x}}=-\frac{K}{2 \epsilon_{x}} F_{(1)}\left\langle\mathcal{I}_{2,0}\right\rangle, \\
\frac{\partial \tilde{V}_{n r, s c}}{\partial \tilde{a}_{y}}=-\frac{K}{2 \epsilon_{x}} F_{(1)}\left\langle\mathcal{I}_{0,2}\right\rangle \epsilon_{r},
\end{gathered}
$$

and the difference of tune between the particle at $\tilde{a}_{x}, \tilde{a}_{y} \simeq 0$ and the bare tune is

$$
\begin{aligned}
\Delta Q_{x} & =-\frac{K}{2} F_{(1)}\left\langle\mathcal{I}_{2,0}\right\rangle \frac{1}{\epsilon_{x}} R, \\
\Delta Q_{y} & =-\frac{K}{2} F_{(1)}\left\langle\mathcal{I}_{0,2}\right\rangle \frac{1}{\epsilon_{y}} R .
\end{aligned}
$$

Next, by considering a smooth lattice, i.e., with beta functions not oscillating too wildly, we find

$$
\begin{aligned}
& \left\langle\mathcal{I}_{2,0}\right\rangle \simeq \frac{2 \bar{\sigma}_{x}}{\bar{\sigma}_{x}+\bar{\sigma}_{y}}=\frac{2 \bar{\sigma}_{x}^{2}}{\bar{\sigma}_{x}\left(\bar{\sigma}_{x}+\bar{\sigma}_{y}\right)}=\frac{R}{Q_{x}} \epsilon_{x} \frac{2}{\bar{\sigma}_{x}\left(\bar{\sigma}_{x}+\bar{\sigma}_{y}\right)}, \\
& \left\langle\mathcal{I}_{0,2}\right\rangle \simeq \frac{2 \bar{\sigma}_{y}}{\bar{\sigma}_{x}+\bar{\sigma}_{y}}=\frac{2 \bar{\sigma}_{y}^{2}}{\bar{\sigma}_{y}\left(\bar{\sigma}_{x}+\bar{\sigma}_{y}\right)}=\frac{R}{Q_{y}} \epsilon_{y} \frac{2}{\bar{\sigma}_{y}\left(\bar{\sigma}_{x}+\bar{\sigma}_{y}\right)} .
\end{aligned}
$$

Substituting, we find

$$
\begin{aligned}
& \Delta Q_{x}=-K F_{(1)} \frac{R^{2}}{Q_{x}} \frac{1}{\bar{\sigma}_{x}\left(\bar{\sigma}_{x}+\bar{\sigma}_{y}\right)}, \\
& \Delta Q_{y}=-K F_{(1)} \frac{R^{2}}{Q_{y}} \frac{1}{\bar{\sigma}_{y}\left(\bar{\sigma}_{x}+\bar{\sigma}_{y}\right)} .
\end{aligned}
$$

For a Gaussian beam $F(v)=-e^{-v / 2}$ and then $F_{(1)}=1 / 2$; therefore, we find

$$
\Delta Q_{x}=-\frac{K}{2} \frac{R^{2}}{Q_{x}} \frac{1}{\bar{\sigma}_{x}\left(\bar{\sigma}_{x}+\bar{\sigma}_{y}\right)},
$$




$$
\Delta Q_{y}=-\frac{K}{2} \frac{R^{2}}{Q_{y}} \frac{1}{\bar{\sigma}_{y}\left(\bar{\sigma}_{x}+\bar{\sigma}_{y}\right)},
$$

which is the standard expression of the incoherent space charge tune shift for circular accelerators. This expression is valid for small $\Delta Q_{\chi} / Q_{\chi 0}$, which is the case in the incoherent space charge regime of concern in this study.

\section{APPENDIX F: CANONICAL EQUATIONS AND STATIONARY POINTS IN THE REDUCED SYSTEM}

The Hamiltonian of the reduced system $C$ reads

$\mathrm{H}^{C}=\frac{4 \rho_{s}}{R} \mathrm{a}^{n_{x} / 2} \mathrm{a}_{y}^{n_{y} / 2} \cos [\Phi] N_{x}+\frac{\Delta_{r 0}}{R} \mathrm{a}+N_{x} \mathrm{~V}(\mathrm{a}, \mathrm{C})$

with $\mathrm{V}(\mathrm{a}, \mathrm{C})=\tilde{V}_{n r}\left[\mathrm{a},\left(N_{y} \mathrm{a}-\mathrm{C}\right) / N_{x}\right]$, and the canonical equations are

$$
\begin{aligned}
& \mathbf{a}^{\prime}=-\frac{\partial \mathrm{H}^{C}}{\partial \Phi}, \\
& \Phi^{\prime}=\frac{\partial \mathbf{H}^{C}}{\partial \mathbf{a}} ;
\end{aligned}
$$

namely,

$$
\begin{gathered}
\mathrm{a}^{\prime}=\frac{4 \rho_{s}}{R} N_{x} \mathrm{a}^{n_{x} / 2} \mathrm{a}_{y}^{n_{y} / 2} \sin (\Phi), \\
\Phi^{\prime}=\frac{2 \rho_{s}}{R} \mathrm{a}^{n_{x} / 2} \mathrm{a}_{y}^{n_{y} / 2}\left(N_{x} \frac{n_{x}}{\mathrm{a}}+N_{y} \frac{n_{y}}{\mathrm{a}_{y}}\right) \cos (\Phi) \\
+\frac{\Delta_{r 0}}{R}+N_{x} \frac{d \mathrm{~V}}{d \mathrm{a}}
\end{gathered}
$$

The stationary point is found solving

$$
\begin{gathered}
0=\frac{4 \rho_{s}}{R} N_{x} \mathrm{a}^{n_{x} / 2} \mathrm{a}_{y}^{n_{y} / 2} \sin (\Phi), \\
0=\frac{2 \rho_{s}}{R} \mathrm{a}^{n_{x} / 2} \mathrm{a}_{y}^{n_{y} / 2}\left(N_{x} \frac{n_{x}}{\mathrm{a}}+N_{y} \frac{n_{y}}{\mathrm{a}_{y}}\right) \cos (\Phi) \\
+\frac{\Delta_{r 0}}{R}+N_{x} \frac{d \mathrm{~V}}{d \mathrm{a}} .
\end{gathered}
$$

The first equation has the solution $\Phi=\pi q$, with $q$ an integer, and the second equation instead becomes

$$
\begin{aligned}
0= & \frac{2 \rho_{s}}{R} \mathrm{a}^{n_{x} / 2} \mathrm{a}_{y}^{n_{y} / 2}\left(N_{x} \frac{n_{x}}{\mathrm{a}}+N_{y} \frac{n_{y}}{\mathrm{a}_{y}}\right)(-1)^{q} \\
& +\frac{\Delta_{r 0}}{R}+N_{x} \frac{d \mathrm{~V}}{d \mathrm{a}} .
\end{aligned}
$$

The value of $q$ should be taken to allow the existence of the stationary point. Once the stationary point $a$ is found, we also find $\mathrm{a}_{y}=\left(N_{y} \mathrm{a}-\mathrm{C}\right) / N_{x}$. By using this solution in the canonical equation generated by the Hamiltonian Eq. (5) and imposing $\tilde{\varphi}_{x}, \tilde{\varphi}_{y}$ to be stationary, we find $t_{x}, t_{y}$. Therefore, we have a relation between $\mathrm{C}$ and $t_{x}$.

\section{Secondary tunes}

We discuss here the secondary tunes in the reduced system. Take a fixed line a, $\Phi=\pi q$ and consider a perturbation of $a, \Phi$ around the fixed line. The evolution of the perturbation reads

$$
\begin{gathered}
\delta \mathbf{a}^{\prime}=-\frac{\partial}{\partial \Phi} \frac{\partial \mathrm{H}^{C}}{\partial \Phi} \delta \Phi, \\
\delta \Phi^{\prime}=\frac{\partial}{\partial \mathrm{a}} \frac{\partial \mathrm{H}^{C}}{\partial \mathrm{a}} \delta \mathrm{a},
\end{gathered}
$$

because on a fixed line $\frac{\partial^{2} \mathrm{H}^{C}}{\partial \mathrm{a} \partial \Phi}=0$. Therefore,

$$
\begin{gathered}
\delta \mathbf{a}^{\prime \prime}=-\left(\frac{\partial}{\partial \Phi} \frac{\partial \mathbf{H}^{C}}{\partial \Phi}\right)^{\prime} \delta \Phi-\left(\frac{\partial}{\partial \Phi} \frac{\partial \mathrm{H}^{C}}{\partial \Phi}\right) \delta \Phi^{\prime} \\
\delta \Phi^{\prime}=\frac{\partial}{\partial \mathbf{a}} \frac{\partial \mathrm{H}^{C}}{\partial \mathrm{a}} \delta \mathrm{a} .
\end{gathered}
$$

Now we use the consequence that on the fixed line

$$
\left(\frac{\partial}{\partial \Phi} \frac{\partial \mathrm{H}^{C}}{\partial \Phi}\right)^{\prime}=0
$$

because a and $\Phi$ are constant. Therefore, the equation of the perturbation $\delta$ a becomes

$$
\delta \mathrm{a}^{\prime \prime}+\omega^{2} \delta \mathrm{a}=0,
$$

with

$$
\omega^{2}=\left(\frac{\partial}{\partial \Phi} \frac{\partial \mathrm{H}^{C}}{\partial \Phi}\right) \frac{\partial}{\partial \mathrm{a}} \frac{\partial \mathrm{H}^{C}}{\partial \mathrm{a}} .
$$

As

$$
\begin{aligned}
& \frac{\partial^{2} \mathrm{H}^{C}}{\partial \Phi^{2}}=-\frac{4 \rho_{s}}{R} \mathrm{a}^{n_{x} / 2} \mathrm{a}_{y}^{n_{y} / 2} \cos [\pi q] N_{x}, \\
& \frac{\partial^{2} \mathrm{H}^{C}}{\partial \mathrm{a}^{2}}=\frac{4 \rho_{s}}{R} \frac{d^{2}}{d \mathrm{a}^{2}}\left(\mathbf{a}^{n_{x} / 2} \mathrm{a}_{y}^{n_{y} / 2}\right) \cos [\pi q] N_{x}+N_{x} \frac{d^{2} \mathrm{~V}}{d \mathrm{a}^{2}},
\end{aligned}
$$

at last we find

$$
\begin{aligned}
\omega^{2}= & -\left[\frac{4 \rho_{s}}{R} \mathbf{a}^{n_{x} / 2} \mathbf{a}_{y}^{n_{y} / 2} \cos [\pi q] N_{x}\right] \\
& \times\left[\frac{4 \rho_{s}}{R} \frac{d^{2}}{d \mathbf{a}^{2}}\left(\mathbf{a}^{n_{x} / 2} \mathbf{a}_{y}^{n_{y} / 2}\right) \cos [\pi q] N_{x}+N_{x} \frac{d^{2} \mathrm{~V}}{d \mathbf{a}^{2}}\right] .
\end{aligned}
$$


If $\omega^{2}$ is negative, the fixed line is unstable; otherwise, $Q_{2}=$ $\omega R$ is the secondary tune. For a Gaussian beam, the secondorder derivative $d^{2} \mathrm{~V} / d \mathrm{a}^{2}$ does not change sign; then when space charge is present and for weak resonances we can always find a value of $q$ that makes $\omega^{2}>0$.

\section{APPENDIX G: THE FORM OF THE FIXED LINES}

On a fixed line, the variables $\tilde{\varphi}_{x}$ and $\tilde{\varphi}_{y}$ are constant on the system of coordinates defined by the transformation characterized by $t_{x}$ : This is because $t_{x}$ is obtained with the procedure described in Appendix F. In addition, $\tilde{\varphi}_{x}$ and $\tilde{\varphi}_{y}$ satisfy the following relation:

$$
N_{x} \tilde{\varphi}_{x}+N_{y} \tilde{\varphi}_{y}+\alpha=\pi q,
$$

while $\tilde{a}_{x}=\mathrm{a}$ and $\tilde{a}_{y}=\mathrm{a}_{y}$ are constant. Let us now express this solution in the laboratory frame. We use the coordinate transformation

$$
\begin{gathered}
\hat{a}_{x}=\tilde{a}_{x}, \\
\hat{a}_{y}=\tilde{a}_{y}, \\
\hat{\varphi}_{x}=\tilde{\varphi}_{x}-t_{x} \frac{2 \pi \Delta_{r 0}}{L} s, \\
\hat{\varphi}_{y}=\tilde{\varphi}_{y}-t_{y} \frac{2 \pi \Delta_{r 0}}{L} s,
\end{gathered}
$$

with $N_{x} t_{x}+N_{y} t_{y}=1$. Therefore, in the laboratory frame we find

$$
\begin{gathered}
\hat{\chi}=\sqrt{\beta_{\chi} \hat{a}_{\chi}} \cos \left(\Psi_{\chi}+\tilde{\varphi}_{\chi}\right), \\
\hat{p}_{\chi}=-\epsilon_{x} \frac{\alpha_{\chi}}{\sqrt{\beta_{\chi}}} \sqrt{\hat{a}_{\chi}} \cos \left(\Psi_{\chi}+\tilde{\varphi}_{\chi}\right) \\
-\epsilon_{x} \frac{1}{\sqrt{\beta_{\chi}}} \sqrt{\hat{a}_{\chi}} \sin \left(\Psi_{\chi}+\tilde{\varphi}_{\chi}\right),
\end{gathered}
$$

where we define for convenience a modified phase advance $\Psi_{\chi}(s)=\psi_{\chi}(s)-t_{\chi}\left(2 \pi \Delta_{r 0} / L\right) s$. The Poincaré surface of the section at $s=0$ is obtained writing the particle coordinates as a function of the turn number. At the $n$th turn, we find

$$
\begin{gathered}
\hat{\chi}=\sqrt{\beta_{\chi} \hat{a}_{\chi}} \cos \left(2 \pi \mathcal{Q}_{\chi} n+\tilde{\varphi}_{\chi}\right), \\
\hat{p}_{\chi}=-\epsilon_{x} \frac{\alpha_{\chi}}{\sqrt{\beta_{\chi}}} \sqrt{\hat{a}_{\chi}} \cos \left(2 \pi \mathcal{Q}_{\chi} n+\tilde{\varphi}_{\chi}\right) \\
-\epsilon_{x} \frac{1}{\sqrt{\beta_{\chi}}} \sqrt{\hat{a}_{\chi}} \sin \left(2 \pi \mathcal{Q}_{\chi} n+\tilde{\varphi}_{\chi}\right),
\end{gathered}
$$

where we define the modified tunes $\mathcal{Q}_{\chi}=\Psi_{\chi}(L) / 2 \pi=$ $Q_{\chi}-t_{\chi} \Delta_{r 0}$. These modified tunes have the property $N_{x} \mathcal{Q}_{x}+N_{y} \mathcal{Q}_{y}=m$. Hence,
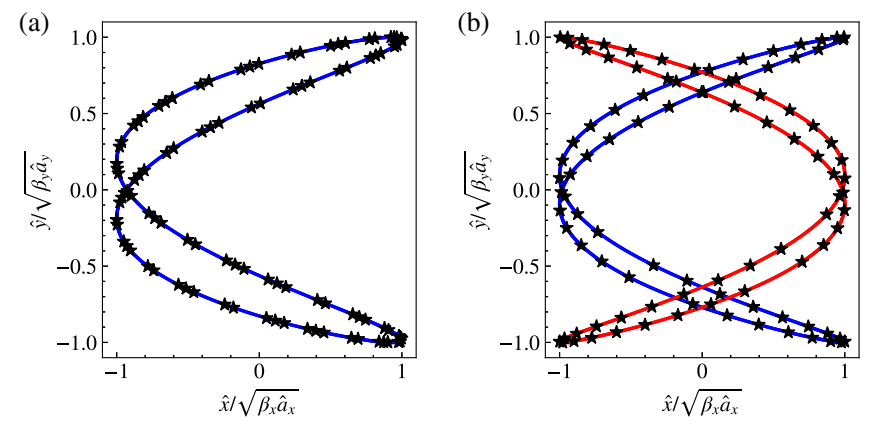

FIG. 7. In (a) is shown an example of a fixed line for $N_{x}=1$, $N_{y}=2$, and $m=19$. The blue curve corresponding to $l=0$ is the theoretical curve, and the black markers signal the location of the beam during 100 turns. (b) shows the case with $N_{x}=2$, $N_{y}=4$, and $m=19$ : Here in the plane $x-y$ there are two distinct parametrizing curves (blue for $l=0$ and red for $l=1$ ).

$$
\begin{gathered}
\mathcal{Q}_{y}=\frac{1}{N_{y}}\left(-N_{x} \mathcal{Q}_{x}+m\right), \\
\tilde{\varphi}_{y}=\frac{1}{N_{y}}\left(-N_{x} \tilde{\varphi}_{x}-\alpha+\pi q\right),
\end{gathered}
$$

and $\tilde{\varphi}_{x}$ is an arbitrary phase determined by the initial condition. Equations (G8)-(G11) are the theoretical description of the Poincare surface of the section for a fixed line of any order (for sum resonances). A possible parameterization of this surface has the form

$$
\begin{gathered}
\hat{x}=\sqrt{\beta_{x} \hat{a}_{x}} \cos \left(-N_{y} t+\frac{2 \pi l}{N_{x}}+\frac{-\alpha+\pi q}{N_{x}}\right), \\
\hat{y}=\sqrt{\beta_{y} \hat{a}_{y}} \cos \left(N_{x} t\right),
\end{gathered}
$$

with $l$ an integer in $0 \leq l<N_{x}$, and $0 \leq t<2 \pi /$ $\operatorname{gcd}\left(N_{x}, N_{y}\right)$, being $\operatorname{gcd}$ the greatest common divisor. Similar results hold for $\hat{p}_{\chi}$. Equations (G12) and (G13) define for each $l$ a closed curve, and according to the coefficients $N_{x}, N_{y}$, and $m$ the parameterization of the Poincaré surface of the section may require several of these maps (each characterized by a specific $l$ ). For the case of $N_{x}=1, N_{y}=2$, and $m=19$, one map suffices to parameterize the fixed line as presented in Ref. [16]. For convenience, we show this feature in Fig. 7(a). Figure 7(b) shows instead the case with $N_{x}=2, N_{y}=4$, and $m=19$ : Here the fixed line appears split into two closed curves, because $N_{x}$ and $N_{y}$ are multiples of 2 , but $m=19$ and $N_{x}=2$ are coprime.

\section{APPENDIX H: STATIONARY POINTS IN THE ABSENCE OF DETUNING}

In the absence of detuning, the equation of the stationary point reads 
$0=\frac{2 \rho_{s}}{R} \mathrm{a}^{n_{x} / 2} \mathrm{a}_{y}^{n_{y} / 2}\left(N_{x} \frac{n_{x}}{\mathrm{a}}+N_{y} \frac{n_{y}}{\mathrm{a}_{y}}\right)(-1)^{q}+\frac{\Delta_{r 0}}{R}$.

The condition for the existence of a solution is $-(-1)^{q} \Delta_{r 0}>0$, and through straightforward algebra, recalling that $\mathrm{a}=\tilde{a}_{x}$ and $\mathrm{a}_{y}=\tilde{a}_{y}$ we find that Eq. $(\mathrm{H} 1)$ admits the infinite set of solutions

$$
\begin{aligned}
& \tilde{a}_{x}=A t_{x}^{\left(2-n_{y}\right) /\left(n_{s}-2\right)} t_{y}^{n_{y} /\left(n_{s}-2\right)}, \\
& \tilde{a}_{y}=\frac{n_{y}}{n_{x}} A t_{x}^{n_{x} /\left(n_{s}-2\right)} t_{y}^{\left(-n_{x}+2\right) /\left(n_{s}-2\right)},
\end{aligned}
$$

with

$$
A=\left[\frac{\left|\Delta_{r 0}\right|}{2 \rho_{s} n_{x}}\left(\frac{n_{x}}{n_{y}}\right)^{n_{y} / 2}\right]^{2 /\left(n_{s}-2\right)}
$$

and $n_{s}=n_{x}+n_{y}$ with $N_{x} t_{x}+N_{y} t_{y}=1$. The secondary frequency is given by Eq. (F13), which in the absence of detuning yields

$$
\omega^{2}=\left(\frac{\left|\Delta_{r 0}\right|}{R}\right)^{2} \frac{2}{n_{y}}\left[\left(\frac{n_{y}}{n_{x}}+1\right)\left(N_{x} t_{x}\right)^{2}-2 N_{x} t_{x}+1-\frac{n_{y}}{2}\right]
$$

with $0 \leq N_{x} t_{x} \leq 1$. For the case $n_{x}=N_{x}=1$ and $n_{y}=$ $N_{y}=2$ we find the secondary tune

$$
Q_{2}=\left|\Delta_{r 0}\right| \sqrt{t_{x}\left(3 t_{x}-2\right)}
$$

when $2 / 3<t_{x} \leq 1$; if instead $0<t_{x}<2 / 3$, the fixed line is unstable. (The red and the blue markers of Fig. 2).

\section{APPENDIX I: THE FUNCTION $\mathcal{V}_{\text {sc }}(\mathbf{a}, \mathrm{C})$}

In the reduced system $C$, the dynamics is described by two conjugate dynamical variables. The canonical equations now allow finding the fixed lines in the presence of detuning. The main feature of the reduced system $C$ is that the dynamical variables $a$ and $a_{y}$ satisfy the equality $N_{y} \mathrm{a}=N_{x} \mathrm{a}_{y}+\mathrm{C}$. The same equality holds for the fixed lines. The slowly varying nonresonant potential reads

$$
\mathrm{V}(\mathrm{a}, \mathrm{C})=\tilde{V}_{n r}\left(\mathrm{a}, \frac{N_{y} \mathrm{a}-\mathrm{C}}{N_{x}}\right)
$$

with the domain of a defined by the simultaneous condition $a_{y} \geq 0$ and $a \geq 0$. We next use this definition in Eq. (D6) for the space charge potential Eq. (3). Observing that $t$ in Eq. (3) has dimension $[t] \equiv\left[\mathrm{m}^{2}\right]$, we perform the change of variable $t=\sigma_{y}^{2} \xi$ and find that the space charge slowly varying potential in the system $C$ reads

$$
\begin{aligned}
\mathrm{V}_{\mathrm{sc}}= & -\frac{K}{\epsilon_{x}} \frac{1}{2 \pi} \int_{0}^{2 \pi} d x \frac{1}{2 \pi} \int_{0}^{2 \pi} d y \frac{1}{L} \int_{0}^{L} d s \int_{0}^{\infty} d \xi \\
& \times \frac{F\left[\hat{T}_{n r}(\xi)\right]-F(0)}{\left(\sigma_{r}^{2}+\xi\right)^{1 / 2}(1+\xi)^{1 / 2}},
\end{aligned}
$$

where

$$
\hat{T}_{n r}(\xi)=\frac{\cos ^{2}(x) \sigma_{r}^{2}}{\sigma_{r}^{2}+\xi} \mathrm{a}+\frac{\cos ^{2}(y)}{1+\xi} \epsilon_{r} \mathrm{a}_{y}
$$

and with $\sigma_{r}=\sigma_{x} / \sigma_{y}=\sqrt{\epsilon_{r} \beta_{x}(s) / \beta_{y}(s)}$ the beam aspect ratio, an $s$-dependent function which depends on $\epsilon_{r}$ and the accelerator optical functions.

The potential $\mathrm{V}_{\mathrm{sc}}$ is used in the canonical equations (12), where it takes the form

$$
\begin{aligned}
N_{x} \frac{d \mathrm{~V}_{\mathrm{sc}}}{d \mathrm{a}}= & -\frac{K}{\epsilon_{x}} \frac{1}{2 \pi} \int_{0}^{2 \pi} d x \frac{1}{2 \pi} \int_{0}^{2 \pi} d y \\
& \times \frac{1}{L} \int_{0}^{L} d s \int_{0}^{\infty} d \xi \frac{F^{\prime}\left[\hat{T}_{n r}(\xi)\right]}{\left(\sigma_{r}^{2}+\xi\right)^{1 / 2}(1+\xi)^{1 / 2}} \\
& \times\left[\frac{N_{x} \cos ^{2}(x) \sigma_{r}^{2}}{\sigma_{r}^{2}+\xi}+\frac{N_{y} \cos ^{2}(y)}{1+\xi} \epsilon_{r}\right] .
\end{aligned}
$$

We recall that the function $F(t)$ originates the particle density expressed in Eq. (A1) and that we consider beam distributions having the maximum at $t=0$, that is, $F^{\prime}(0) \geq F^{\prime}(t) \geq 0$ for $t \geq 0$. Therefore, we also have $F^{\prime}(0) \geq F^{\prime}\left[\hat{T}_{n r}(\xi)\right]$, which implies that

$N_{x} \frac{d \mathrm{~V}_{\mathrm{sc}}}{d \mathrm{a}} \geq-N_{x} K \frac{F_{(1)}}{2 \epsilon_{x}}\left\langle\mathcal{I}_{2,0}\right\rangle-N_{y} K \frac{F_{(1)}}{2 \epsilon_{y}}\left\langle\mathcal{I}_{0,2}\right\rangle=\frac{\mathcal{D}_{r, s c}}{R}$,

where we defined for convenience the quantity

$$
\mathcal{D}_{r, s c}=N_{x} \Delta Q_{x}+N_{y} \Delta Q_{y}
$$

with $\Delta Q_{x}, \Delta Q_{y}$ the incoherent space charge tune shifts discussed in Appendix E. In addition, for a Gaussian distribution we find that for $a \rightarrow \infty$ it is

$$
N_{x} \frac{d \mathrm{~V}_{\mathrm{sc}}}{d \mathrm{a}} \rightarrow 0
$$

Therefore, we find the general result that

$$
\frac{\mathcal{D}_{r, s c}}{R} \leq N_{x} \frac{d \mathrm{~V}_{\mathrm{sc}}}{d \mathrm{a}} \leq 0 .
$$

Equation (I3) allows one to retrieve the perveance $K$ as

$$
K=-\frac{2 \epsilon_{x} \mathcal{D}_{r, s c}}{R F_{(1)}\left(N_{x}\left\langle\mathcal{I}_{2,0}\right\rangle+N_{y} \epsilon_{r}\left\langle\mathcal{I}_{0,2}\right\rangle\right)},
$$


and therefore it becomes very convenient to define the normalized potential

$$
\begin{aligned}
\mathcal{V}_{\mathrm{sc}}= & \frac{2}{F_{(1)}\left\langle N_{x} \mathcal{I}_{2,0}+N_{y} \mathcal{I}_{0,2} \epsilon_{r}\right\rangle} \\
& \times \frac{1}{2 \pi} \int_{0}^{2 \pi} d x \frac{1}{2 \pi} \int_{0}^{2 \pi} d y \frac{1}{L} \int_{0}^{L} d s \int_{0}^{\infty} d \xi \\
& \times \frac{F\left[\hat{T}_{n r}(\xi)\right]-F(0)}{\left(\sigma_{r}^{2}+\xi\right)^{1 / 2}(1+\xi)^{1 / 2}} .
\end{aligned}
$$

This formulation of the slowly varying potential shows that $\mathcal{V}_{\mathrm{sc}}$ has the interesting property to depend only on the beam emittance ratio $\epsilon_{r}$ and the optical functions ratio. As in the incoherent space charge regime the optical functions are, in good approximation, only slightly affected by the Coulomb forces, it follows that for beams with equal $\epsilon_{r}$ the normalized space charge potential $\mathcal{V}_{\text {sc }}$ has in good approximation the same value at the same a. Therefore, $\mathcal{V}_{\mathrm{sc}}$ becomes a purely geometric function. In conclusion, the nonresonant potential is written in terms of the normalized nonresonant potential as

$$
\mathrm{V}_{\mathrm{sc}}=\frac{\mathcal{D}_{r, s c}}{R} \mathcal{V}_{\mathrm{sc}},
$$

where $\mathcal{V}_{\text {sc }}$ for a Gaussian distribution has the useful feature that

$$
0 \leq N_{x} \frac{d \mathcal{V}_{\text {sc }}}{d \mathrm{a}} \leq 1
$$

and it depends purely on the beam size ratio $\sigma_{r}$. In particular, if $\mathrm{C}=0$ and $\mathrm{a} \rightarrow 0$, we find

$$
\mathcal{V}_{\mathrm{sc}}=\frac{\left\langle\mathcal{I}_{2,0} \mathrm{a}+\mathcal{I}_{0,2} \epsilon_{r} \mathrm{a}_{y}\right\rangle}{\left\langle N_{x} \mathcal{I}_{2,0}+N_{y} \mathcal{I}_{0,2} \epsilon_{r}\right\rangle} .
$$

[1] M. Reiser, Theory and Design of Charged Particle Beams (Wiley-VCH, Weinheim, 2004), pp. 184-187.

[2] R. C. Davidson, Physics of Nonneutral Plasmas (AddisonWesley, Redwood City, 1990), p. 59.

[3] I. Hofmann, Stability of anisotropic beams with space charge, Phys. Rev. E 57, 4713 (1998).

[4] I. Hofmann, G. Franchetti, J. Qiang, and R. Ryne, in Proceedings of the 29th ICFA Advanced Beam Dynamics Workshop on Beam Halo Dynamics, Diagnostics, and Collimation HALO 03 (AIP, New York, 2003), Vol. 693, p. 65 .

[5] F. Kesting and G. Franchetti, Propagation of numerical noise in particle-in-cell tracking, Phys. Rev. Accel. Beams 18, 114201 (2015).
[6] J. Struckmeier, Stochastic effects in real and simulated charged particle beams, Phys. Rev. ST Accel. Beams 3, 034202 (2000).

[7] O. Boine-Frankenheim, I. Hofmann, J. Struckmeier, and S. Appel, Artificial collisions, entropy and emittance growth in computer simulations of intense beams, Nucl. Instrum. Methods Phys. Res., Sect. A 770, 164 (2015).

[8] I. Hofmann and O. Boine-Frankenheim, Grid dependent noise and entropy growth in anisotropic $3 \mathrm{~d}$ particle-in-cell simulation of high intensity beams, Phys. Rev. Accel. Beams 17, 124201 (2014).

[9] Ji Qiang, Symplectic particle-in-cell model for spacecharge beam dynamics simulation, Phys. Rev. Accel. Beams 21, 054201 (2018).

[10] R. Hagedorn, CERN Report No. CERN 57-1, 1957.

[11] A. Schoch, CERN Report No. CERN 57-23, 1958.

[12] R. Tomás, M. Bai, R. Calaga, W. Fischer, A. Franchi, and G. Rumolo, Measurement of global and local resonance terms, Phys. Rev. ST Accel. Beams 8, 024001 (2005).

[13] A. Bazzani, E. Todesco, G. Turchetti, and G. Servizi, Report No. CERN 94-02, 1994.

[14] E. Forest, From Tracking Code to Analysis (Springer, Japan, 2016).

[15] F. Schmidt, DESY HERA Report No. 8802, 1988 (to be published).

[16] G. Franchetti and F. Schmidt, Extending the NonlinearBeam-Dynamics Concept of 1D Fixed Points to 2D Fixed Lines, Phys. Rev. Lett. 114, 234801 (2015).

[17] G. Franchetti and F. Schmidt, arXiv:1504.04389.

[18] C. J. A. Corsten and H. L. Hagedoorn, Stopband widths in non-linear sum resonances, Nucl. Instrum. Methods 216, 25 (1983).

[19] S. Ohnuma, Comments on Stable Motions in Nonlinear Coupled Resonances, IEEE Trans. Nucl. Sci. NS-28, 2491 (1981).

[20] E. Todesco, Analysis of resonant structures of fourdimensional symplectic mappings, using normal forms, Phys. Rev. E 50, R4298 (1994).

[21] M. Gemmi and E. Todesco, Stability and geometry of third-order resonances in four-dimensional symplectic mappings, Celest. Mech. Dyn. Astron. 67, 181 (1997).

[22] G. Franchetti, S. Gilardoni, A. Huschauer, F. Schmidt, and R. Wasef, Space charge effects on the third order coupled resonance, Phys. Rev. Accel. Beams 20, 081006 (2017).

[23] G. Turchetti, Hamiltonian maps and normal forms for intense beams, Nucl. Instrum. Methods Phys. Res., Sect. A 561, 151 (2006).

[24] G. Guignard, CERN Report No. CERN 78-11, 1978.

[25] J. A. Sanders and F. Verhulst, Averaging Methods in Nonlinear Dynamical Systems (Springer-Verlag, Berlin, 1985).

[26] S. Y. Lee, Fundamental Limit of Nonscaling Fixed-Field Alternating-Gradient Accelerators, Phys. Rev. Lett. 97, 104801 (2006).

[27] F. J. Sacherer, RMS envelope equations with space charge, IEEE Trans. Nucl. Sci. 18, 1105 (1971). 